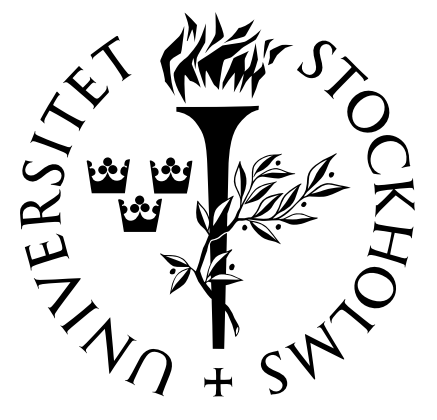

Mathematical Statistics

Stockholm University

\title{
An epidemic model with infector and exposure dependent severity
}

\author{
Frank Ball \\ Tom Britton
}

Research Report 2008:9

ISSN 1650-0377 


\section{Postal address:}

Mathematical Statistics

Dept. of Mathematics

Stockholm University

SE-106 91 Stockholm

Sweden

\section{Internet:}

http://www.math.su.se/matstat 


\title{
An epidemic model with infector and exposure dependent severity
}

\author{
Frank Ball \\ Tom Britton *
}

October 2008

\begin{abstract}
A stochastic epidemic model allowing for both mild and severe infectives is defined, where an infective can become severe directly upon infection or if additionally exposed to infection. It is shown that, assuming a large community, the initial phase of the epidemic may be approximated by a suitable branching process and that the main part of an epidemic that becomes established admits a law of large numbers and a central limit theorem, leading to a normal approximation for the final outcome of such an epidemic. Effects of vaccination prior to an outbreak are studied and the critical vaccination coverage, above which only small outbreaks can occur, is derived. The results are illustrated by simulations that demonstrate that the branching process and normal approximations work well for finite communities, and by numerical examples showing that the final outcome may be close to discontinuous in certain model parameters and that the fraction mildly infected may actually increase as an effect of vaccination.
\end{abstract}

Key words: Stochastic epidemic, final size, basic reproduction number, severity, exposure, vaccination.

*Postal address: Mathematical Statistics, Stockholm University, SE-106 91, Sweden. E-mail: tomb@math.su.se. 



\title{
An epidemic model with infector and exposure dependent severity
}

\author{
Frank Ball ${ }^{1}$ and Tom Britton ${ }^{b}$
}

${ }^{a}$ School of Mathematical Sciences, University of Nottingham, University Park, Nottingham NG7 2RD, UK.

${ }^{b}$ Department of Mathematics, Stockholm University, SE-106 91 Stockholm, Sweden.

\begin{abstract}
A stochastic epidemic model allowing for both mild and severe infectives is defined, where an infective can become severe directly upon infection or if additionally exposed to infection. It is shown that, assuming a large community, the initial phase of the epidemic may be approximated by a suitable branching process and that the main part of an epidemic that becomes established admits a law of large numbers and a central limit theorem, leading to a normal approximation for the final outcome of such an epidemic. Effects of vaccination prior to an outbreak are studied and the critical vaccination coverage, above which only small outbreaks can occur, is derived. The results are illustrated by simulations that demonstrate that the branching process and normal approximations work well for finite communities, and by numerical examples showing that the final outcome may be close to discontinuous in certain model parameters and that the fraction mildly infected may actually increase as an effect of vaccination.
\end{abstract}

Keywords: stochastic epidemic, final size, basic reproduction number, severity, exposure, vaccination.

\section{Introduction}

For several infectious diseases infected people get different severity of the disease and the degree of severity may also affect the ability to spread the disease further. Examples of such diseases are dengue fever (Mangada and Igarashi [1]), measles (Morley and Aaby [2]) and varicella (Parang and Archama [3]). In the present paper we analyse a stochastic epidemic model allowing for different severities. More precisely the model has two degrees of severity,

\footnotetext{
${ }^{1}$ Corresponding author. Tel.: +44-115 951 4969; fax +44-115 9514951.

E-mail addresses: frank.ball@nottingham.ac.uk (F. Ball), tom.britton@math.su.se (T. Britton)
} 
mild and severe, and the degree of severity affects both the rate of contact an infective has with other individuals and the probabilities that the contacted individuals becomes mildly or severely infected. Additional to an individual becoming severely infected directly upon infection, the model also allows for mild cases to become severe if exposed further to the disease. We also define a vaccine response model where the vaccine may reduce susceptibility, infectivity and/or the length of the infectious period.

As the community size increases we show that the initial phase of an epidemic with few initial infectives may be approximated by a suitable branching process (in which individuals cannot change from mild to severe!) and the basic reproduction number $R_{0}$ is derived; $R_{0}>1$ indicates that a significant fraction of the community may become infected, i.e. that a major epidemic may occur. Also assuming that the community size tends to infinity, a law of large numbers and central limit theorem for the final number of mild and severely infected are obtained for the situation where there is a major outbreak. We also sketch the corresponding results for the case that a fraction $v$ of the community are vaccinated prior to introduction of the disease, and derive the critical vaccination coverage $v_{c}$ needed to surely prevent a major outbreak.

The results are illustrated by numerical examples. We show by means of simulations that the branching approximation, and the central limit theorem for the final outcome in the event of a major outbreak, work satisfactorily as an approximation in a finite community. We also illustrate how the limiting final proportion infected depends on certain model parameters and how the proportion is reduced as a function of $v$ for three specific vaccine response cases denoted all-or-nothing, leaky and non-random.

Even though deterministic limits for the final proportion infected are obtained, they are not very explicit in the parameters; for this reason we give some more explicit bounds. We also show that the final proportion infected can be close to discontinuous in certain model parameters. Another observation is that the proportion mildly infected need not be monotonically decreasing in $v$, the fraction vaccinated, implying that vaccinating more individuals can have the effect that more people become mildly infected. Finally, we show that even though a vaccine response reducing infectivity by a certain factor gives the same reproduction number as if instead the length of the infectious period is reduced by the same factor, the two vaccine responses do not give identical fractions ultimately infected.

In Section 2, the epidemic and vaccine response models are defined. In Section 3, we present our main results together with short heuristic motivations for them. The illustrative examples are given in Section 4 and formal proofs for most of our results appear in Section 5. The paper ends with a short discussion in Section 6. 


\section{The epidemic model and vaccination response model}

\subsection{The infector-exposure dependent severity epidemic model}

We now define the infector-exposure dependent severity (IEDS) epidemic model. In the model there are two types of disease severity, mild and severe, and an individual may become mild or severe upon infection, with probabilities of the two alternatives depending on whether the individual was infected by a mildly or severely infectious individual. Additionally, a mildly infectious individual (denoted mild infective) may turn into a severely infectious individual (severe infective) if he/she is subsequently exposed to the infection.

Consider a closed homogeneously mixing community having initially, i.e. at time $t=0, n$ susceptibles, $m_{\mathrm{M}}$ mild infectives and $m_{\mathrm{S}}$ severe infectives. Mild infectives have close contact with others, chosen uniformly at random from the $n$ initial susceptibles, at rate $\lambda_{\mathrm{M}}$ and severe infectives have close contact with others, chosen uniformly at random from the $n$ initial susceptibles, at rate $\lambda_{\mathrm{S}}$. A close contact by a mild infective with a susceptible results in the contacted susceptible becoming mildly infective with probability $p_{\mathrm{MM}}$ and severely infected with the complimentary probability $p_{\mathrm{MS}}=1-p_{\mathrm{MM}}$. Similarly, a close contact by a severe infective with a susceptible results in the latter becoming mildly infected with probability $p_{\mathrm{SM}}$ and severely infected with the remaining probability $p_{\mathrm{SS}}=1-p_{\mathrm{SM}}$. Further, a mild infective "reinfects" any given mild infective at rate $\lambda_{\mathrm{M}} \alpha_{\mathrm{M}} / n$, and a severe infective "reinfects" any given mild infective at rate $\lambda_{\mathrm{S}} \alpha_{\mathrm{S}} / n$, where "reinfects" means that the contacted mild infective becomes a severe infective. Finally, mild infectives recover and become immune at rate $\gamma_{\mathrm{M}}$ and severe infectives recover and become immune at rate $\gamma_{\mathrm{S}}$, implying that (without reinfection) the infectious periods are assumed to be exponentially distributed. All contact processes, removal processes and uniform 'selections' are assumed to be mutually independent. For $t \geq 0$, let $\boldsymbol{X}^{(n)}(t)=\left(X(t), Y_{\mathrm{M}}(t), Y_{\mathrm{S}}(t), Z_{\mathrm{M}}(t), Z_{\mathrm{S}}(t)\right)$, where $X(t), Y_{\mathrm{M}}(t), Y_{\mathrm{S}}(t), Z_{\mathrm{M}}(t)$ and $\left.Z_{\mathrm{S}}(t)\right)$ denote respectively the number of susceptibles, the number of mild infectives, the number of severe infectives, the number of recovered from the mild state and the number of recovered from the severe state at time $t$.

The present model has several other models studied earlier as special cases. If for instance $\alpha_{\mathrm{M}}=\alpha_{\mathrm{S}}=0$ there is no possibility to shift infectious state and we then have the infectordependent severity (IDS) model investigated by Ball and Britton [4]. Two special cases (assuming $\alpha_{\mathrm{M}}=\alpha_{\mathrm{S}}=0$ ) are where $p_{\mathrm{MM}}=p_{\mathrm{SM}}=1$, when all infected individuals become mildly infected, or the opposite, $p_{\mathrm{MM}}=p_{\mathrm{SM}}=0$, when all become severely infected. We then have the general stochastic epidemic (see, for example, Bailey [5], Chapter 6) with (individual to individual) infection rate $n \lambda_{\mathrm{M}}$ and removal rate $\gamma_{\mathrm{M}}$ (or $n \lambda_{\mathrm{S}}$ and $\gamma_{\mathrm{S}}$, respectively), except possibly for the initial infectives. Another special case here is when $p_{\mathrm{MM}}=p_{\mathrm{SS}}=1$, so the type of an infected individual is always the same as that of his/her infector. The model then becomes the so-called competing epidemic model studied by Kendall and Saunders [6], Scalia-Tomba and Svensson [7] and Scalia-Tomba [8]. 
If instead $p_{\mathrm{MS}}=p_{\mathrm{SS}}=0$, all infected individuals first become mildly infected but may later become severely infected if additionally exposed to infection. We then have a model that is very similar to the exposure dependent severity (EDS) model studied by Ball and Britton [9]. The present model differs from the EDS model in that if a mildly infectious individual is reinfected then he/she immediately stops being a mild infective and becomes a severe infective. In the EDS model, the mild and severe infectious periods can overlap, or be completely disjoint, and the severe state has more the interpretation of being an additional infection pressure.

Another special case is when $\lambda_{\mathrm{M}}=\lambda_{\mathrm{S}}(=\lambda)$ and $\gamma_{\mathrm{M}}=\gamma_{\mathrm{S}}(=\gamma)$. Then there is no difference in how how much infectivity the two severities generate, so the total number of infected behaves like the general stochastic epidemic model with infection and removal rates $n \lambda$ and $\gamma$, respectively.

\subsection{A vaccination response model}

Suppose that, prior to the outbreak, a fraction $v$ of the susceptible population is vaccinated. We now describe the vaccine response model. Each individual that is vaccinated has an independent random vaccine response described by a three dimensional random vector $(A, B, \Theta)$, where $A \geq 0$ is associated with the susceptibility and $B \geq 0$ and $\Theta \geq 0$ are associated with the infectivity and infectious period in the event that the individual does become infected. In general, the random variables $A, B$ and $\Theta$ may be correlated, and typically they are all bounded above by 1 (unless the vaccine increases susceptibility/infectivity). More specifically, an individual who has vaccine response $(a, b, \theta)$ has relative risk $a$ of becoming infected (either mildly or severely) from a contact, when compared to an unvaccinated individual. If the vaccinated person becomes infected, the close contact rate is reduced by the factor $b^{\theta}$, compared to an unvaccinated infectious individual of the same type. Further, the recovery rate of the individual is changed, compared to an unvaccinated infective of the same type, by the factor $b^{\theta-1}$ (so when $b<1$ and $\theta<1$ the recovery rate is increased implying a shorter infectious period, due to the vaccine). Finally, the vaccination has no effect on the rate at which an individual switches from being mildly infected to severely infected.

The meaning of $a$ is hence the relative susceptibility of the vaccinee, $b$ is the relative expected infectious force exerted by the vaccinee if infected (compared to an unvaccinated individual) and $\theta$ quantifies if the reduction in infectious force is due to a lower infectivity while infectious and/or a shorter infectious period. The case $\theta=1$ corresponds to only lower infectivity but unchanged infectious period, and $\theta=0$ is the other extreme where the infectivity is unchanged but the infectious period is reduced. If $P(\Theta=1)=1$, so the infectious period of an infected individual is unchanged by vaccination, then the vaccine response model reduces to the one introduced by Becker and Starczak [10].

There are various measures of vaccine efficacy that can be defined for this vaccine action model, according to whether reduction in susceptibility, infectivity or susceptibility-infectivity is of interest, cf. Becker et al. [11]. The one used in this paper is $V E_{S I}=1-E[A B]$. Note 
that for a single-type epidemic among a homogeneously mixing population, if a proportion $v$ of the population are vaccinated in advance of an outbreak then, under the present vaccine response model, the basic reproduction number $R_{0}$ is reduced to $\left(1-v V E_{S I}\right) R_{0}$ (cf. Becker and Starczak [10]).

In what follows we study two specific forms of vaccine response. The first one is where $(A, B, \Theta)=(0, \cdot, \cdot)$ with probability $e$, and $(A, B, \Theta)=(1,1,1)$ with the remaining probability $1-e$. (Note that whenever $A=0$ a vaccinated individual cannot be infected, so the reduction in infectivity and/or duration of infectious period, if infected, becomes irrelevant). This vaccine response, defined in Smith et al. [12], is commonly known as the all-or-nothing vaccine response (Halloran et al. [13]) and has $V E_{S I}=e$. The other vaccination response treated in the present paper is $(A, B, \Theta)=(a, b, \theta)$ with probability 1 , for some constants $a, b$ and $\theta$, so $V E_{S I}=1-a b$. Following Ball and Lyne [14], we call this vaccine response the nonrandom vaccine response. It is worth noting that, for the non-random vaccine response, when $\theta=1$ the infectivity is reduced to $b$, relative to an unvaccinated individual, and the infectious period is unchanged; and $\theta=0$ implies that the infectivity is unchanged but the average infectious period is reduced to $b$, relative to an unvaccinated individual. In fact, the reduction in infectivity multiplied by the reduction in infectious period equals $b^{\theta} / b^{\theta-1}=b$ which is hence independent of $\theta$. Nevertheless, $\theta$ does have an effect on the outcome of the epidemic, as is illustrated in Section 4. If $b=1$ in the non-random case, then the vaccine response is the so-called leaky response (Halloran et al. [13]). Note that in this case the value of $\theta$ is irrelevant and $V E_{S I}=1-a$, so the efficacy of an all-or-nothing vaccine can be matched by setting $a=1-e$.

\section{Main results and heuristic arguments}

In this section we present large population properties of the IEDS model, first without and then with vaccination. The results are asymptotic assuming the population size tends to infinity. Alongside the results we also give heuristic arguments supporting the results. Proofs to most results are given in Section 5 .

\subsection{Initial stages of the epidemic}

Assume that $n$, the initial number of susceptibles, is large but that the initial number of mild and severe infectives $m_{\mathrm{M}}$ and $m_{\mathrm{S}}$ are small (in the asymptotic setting $n$ tend to infinity whereas $m_{\mathrm{M}}$ and $m_{\mathrm{S}}$ are kept fixed). Then the initial stages of the epidemic can be approximated by a two-type branching process. This follows because it is very unlikely that infective individuals happen to have contact with individuals who have already been contacted, so infectives infect new individuals (more or less) independently of each other; such independence is the fundamental assumption in a branching process. For the same reason, during the early stages 
it is very unlikely that an infective will "reinfect" a mild infective, since the rate at which this happens is proportional to the proportion of the population that are mildly infected, and hence is small during the early stages when $n$ is large. Thus these reinfections do not occur in the approximating branching process, so it is identical to the approximating branching process of the IDS-model (Ball and Britton [4]) in which mildly infected individuals cannot be reinfected to become severely infected.

It follows that the process of infectives in the early stages of the IEDS epidemic model in a large community may be approximated by a two-type branching process. The two types, M and $\mathrm{S}$ (corresponding to mild and severe infectives, respectively), have exponential lifetimes with parameters $\gamma_{\mathrm{M}}$ and $\gamma_{\mathrm{S}}$ (i.e. means $\gamma_{\mathrm{M}}^{-1}$ and $\gamma_{\mathrm{S}}^{-1}$ ), respectively. While alive, M-individuals give birth to M-individuals at rate $\lambda_{\mathrm{M}} p_{\mathrm{MM}}$ and S-individuals at rate $\lambda_{\mathrm{M}} p_{\mathrm{MS}}$, and S-individuals give birth to M-individuals at rate $\lambda_{\mathrm{S}} p_{\mathrm{SM}}$ and S-individuals at rate $\lambda_{\mathrm{S}} p_{\mathrm{SS}}$. The approximating branching process is initiated with $m_{\mathrm{M}} \mathrm{M}$-individuals and $m_{\mathrm{S}}$ S-individuals.

The mean offspring matrix of this branching process is

$$
M=\left[\begin{array}{ll}
\mu_{\mathrm{MM}} & \mu_{\mathrm{MS}} \\
\mu_{\mathrm{SM}} & \mu_{\mathrm{SS}}
\end{array}\right]:=\left[\begin{array}{ll}
\lambda_{\mathrm{M}} p_{\mathrm{MM}} / \gamma_{\mathrm{M}} & \lambda_{\mathrm{M}} p_{\mathrm{MS}} / \gamma_{\mathrm{M}} \\
\lambda_{\mathrm{S}} p_{\mathrm{SM}} \gamma_{\mathrm{S}} & \lambda_{\mathrm{S}} p_{\mathrm{SS}} / \gamma_{\mathrm{S}}
\end{array}\right],
$$

each component being the close-contact rate multiplied by the average length of the infectious period $\left(1 / \gamma_{\mathrm{M}}\right.$ and $1 / \gamma_{\mathrm{M}}$ for mild and severe infectives, respectively). The basic reproduction number $R_{0}$ (e.g. Heesterbeek and Dietz [15]) equals the largest positive eigenvalue of $M$, which can be shown to equal

$$
R_{0}=\frac{1}{2}\left(\mu_{\mathrm{MM}}+\mu_{\mathrm{SS}}+\sqrt{\left(\mu_{\mathrm{MM}}+\mu_{\mathrm{SS}}\right)^{2}+4 \frac{\lambda_{\mathrm{M}} \lambda_{\mathrm{S}}}{\gamma_{\mathrm{M}} \gamma_{\mathrm{S}}}\left(1-p_{\mathrm{MM}}-p_{\mathrm{SS}}\right)}\right) .
$$

It is well-known (e.g. Haccou et al. [16], page 123) that, provided all the elements of $M$ are strictly positive, the branching process has non-zero probability of taking off if and only if $R_{0}>1$.

It is also possible to derive the probability that the epidemic takes off using branching process theory. A mild infective infects new mild cases at the rate $\lambda_{\mathrm{M}} p_{\mathrm{MM}}$ and new severe cases at the rate $\lambda_{\mathrm{M}} p_{\mathrm{MS}}$, during its infectious period which is exponentially distributed having mean $1 / \gamma_{\mathrm{M}}$. Conditional upon the length of the infectious period, the number of infected of the two types are independent and Poisson distributed. Removing the conditioning makes the two random variables dependent. One way to describe their joint distribution is that their sum is geometrically distributed with probability parameter $\gamma_{\mathrm{M}} /\left(\gamma_{\mathrm{M}}+\lambda_{\mathrm{M}}\right)$ (and mean $\lambda_{\mathrm{M}} / \gamma_{\mathrm{M}}$ ) and, given the sum, the number of mildly infected is binomially distributed with number of trials given by this sum and success probability $p_{\mathrm{MM}}$. Let $\left(X_{\mathrm{MM}}, X_{\mathrm{MS}}\right)$ be a bivariate random variable giving the numbers of mild and severe cases created by a typical mild infective and define $\left(X_{\mathrm{SM}}, X_{\mathrm{SS}}\right)$ similarly for a typical severe infective. Then, exploiting the above conditioning yields that the probability generating functions $\rho_{\mathrm{M}}$ and $\rho_{\mathrm{S}}$ of $\left(X_{\mathrm{MM}}, X_{\mathrm{MS}}\right)$ and $\left(X_{\mathrm{SM}}, X_{\mathrm{SS}}\right)$, 
respectively, are

$$
\begin{gathered}
\rho_{\mathrm{M}}\left(s_{1}, s_{2}\right)=E\left(s_{1}^{X_{\mathrm{MM}}} s_{2}^{X_{\mathrm{MS}}}\right)=\frac{\gamma}{\gamma+\lambda_{\mathrm{M}}\left(p_{\mathrm{MM}}\left(1-s_{1}\right)+p_{\mathrm{MS}}\left(1-s_{2}\right)\right)}, \\
\rho_{\mathrm{S}}\left(s_{1}, s_{2}\right)=E\left(s_{1}^{X_{\mathrm{SM}}} s_{2}^{X_{\mathrm{SS}}}\right)=\frac{\gamma}{\gamma+\lambda_{\mathrm{S}}\left(p_{\mathrm{SM}}\left(1-s_{1}\right)+p_{\mathrm{SS}}\left(1-s_{2}\right)\right)} .
\end{gathered}
$$

If the epidemic is initiated by one mild infective, then the epidemic will not take off if and only if every individual that the initial person infects (if any) avoids creating a major outbreak. Let $\pi_{\mathrm{M}}$ denote the probability that the epidemic does not take off given that it is started by one mild infective, and let $\pi_{\mathrm{S}}$ denote the corresponding probability when the epidemic is started by one severe infective. Then the above reasoning (well-known from branching process theory) motivates that $\pi_{\mathrm{M}}$ and $\pi_{\mathrm{S}}$ must solve the equations

$$
\begin{aligned}
& x_{\mathrm{M}}=\sum_{i, j} x_{\mathrm{M}}^{i} x_{\mathrm{S}}^{j} P\left(X_{\mathrm{MM}}=i, X_{\mathrm{MS}}=j\right)=\rho_{\mathrm{M}}\left(x_{\mathrm{M}}, x_{\mathrm{S}}\right)=\frac{\gamma}{\gamma+\lambda_{\mathrm{M}}\left(p_{\mathrm{MM}}\left(1-x_{\mathrm{M}}\right)+p_{\mathrm{MS}}\left(1-x_{\mathrm{S}}\right)\right)}, \\
& x_{\mathrm{S}}=\sum_{i, j} x_{\mathrm{M}}^{i} x_{\mathrm{S}}^{j} P\left(X_{\mathrm{SM}}=i, X_{\mathrm{SS}}=j\right)=\rho_{\mathrm{S}}\left(x_{\mathrm{M}}, x_{\mathrm{S}}\right)=\frac{\gamma}{\gamma+\lambda_{\mathrm{S}}\left(p_{\mathrm{SM}}\left(1-x_{\mathrm{M}}\right)+p_{\mathrm{SS}}\left(1-x_{\mathrm{S}}\right)\right)} .
\end{aligned}
$$

It can be shown that, when $R_{0}>1$ and every element of $M$ is strictly positive, then these equations have a unique solution $\left(\pi_{\mathrm{M}}, \pi_{\mathrm{S}}\right)$ in $[0,1)^{2}$, and these are the probabilities of avoiding a major outbreak when starting with one mild infective, or one severe infective, respectively. If the epidemic is initiated by $m_{\mathrm{M}}$ mild and $m_{\mathrm{S}}$ severe infectives, then the probability that the epidemic takes off equals

$$
P\left(\text { major outbreak } \mid m_{\mathrm{M}}, m_{\mathrm{S}}\right)=1-\pi_{\mathrm{M}}^{m_{\mathrm{M}}} \pi_{\mathrm{S}}^{m_{\mathrm{S}}} .
$$

If $M$ has one or more zero elements the branching process is decomposable (see e.g. Haccou et al. [16], page 26$)$ and $\left(\pi_{\mathrm{M}}, \pi_{\mathrm{S}}\right)$ can be determined by considering an appropriate embedded single-type branching process.

Let $\left(Z_{\mathrm{M}}, Z_{\mathrm{S}}\right)$ denote the ultimate number of individuals ever born in the branching process. Similarly, let $\left(Z_{\mathrm{M}}^{(n)}, Z_{\mathrm{S}}^{(n)}\right)$ be the final number of removed of the two types in the epidemic starting with $n$ susceptible individuals. Then it can be shown that the distribution of $\left(Z_{\mathrm{M}}^{(n)}, Z_{\mathrm{S}}^{(n)}\right)$ converges to that of $\left(Z_{\mathrm{M}}, Z_{\mathrm{S}}\right)$ as $n \rightarrow \infty$; see Corollary 5.2 in Section 5.1, where the stronger, almost sure convergence is proved. The latter is the distribution of the total progeny of a two-type branching process. If $R_{0}>1$ the branching process can grow beyond all limits, thus implying that the distribution of $\left(Z_{\mathrm{M}}, Z_{\mathrm{S}}\right)$ is defective, with strictly positive probability that both random variables are infinite. For the epidemic this corresponds to the case that there is a major outbreak meaning that the number of ultimately infected is of order $n$ rather than of order 1. The size of such an outbreak is described in the next subsection. In Section 4.1 we illustrate how the distinction of minor and major outbreaks in a finite community can be determined using the branching process approximation. 


\subsection{Limiting epidemic process}

Suppose that $n$ is large and assume that the limits $\mu_{\mathrm{M}}:=\lim _{n \rightarrow \infty} m_{\mathrm{M}} / n$ and $\mu_{\mathrm{S}}:=\lim _{n \rightarrow \infty} m_{\mathrm{S}} / n$ exist. As described further in Section 5.2, provided that $\mu_{\mathrm{M}}+\mu_{\mathrm{S}}>0$, the stochastic IEDS model can be approximated by a deterministic model. More precisely, if we let $\overline{\boldsymbol{X}}^{(n)}=$ $\left\{\overline{\boldsymbol{X}}^{(n)}(t): t \geq 0\right\}$, where, for $t \geq 0$,

$$
\overline{\boldsymbol{X}}^{(n)}(t)=\left(\bar{X}^{(n)}(t), \bar{Y}_{\mathrm{M}}^{(n)}(t), \bar{Y}_{\mathrm{S}}^{(n)}(t), \bar{Z}_{\mathrm{M}}^{(n)}(t), \bar{Z}_{\mathrm{S}}^{(n)}(t)\right)=\frac{1}{n}\left(X(t), Y_{\mathrm{M}}(t), Y_{\mathrm{S}}(t), Z_{\mathrm{M}}(t), Z_{\mathrm{S}}(t)\right),
$$

then it can be shown that, as $n$ tends to infinity, this normalised process converges to the solution $\boldsymbol{x}=\{\boldsymbol{x}(t): t \geq 0\}$ of the following set of differential equations

$$
\begin{aligned}
& \frac{d x}{d t}=-\left(\lambda_{\mathrm{M}} y_{\mathrm{M}}+\lambda_{\mathrm{S}} y_{\mathrm{S}}\right) x \\
& \frac{d y_{\mathrm{M}}}{d t}=\left(\lambda_{\mathrm{M}} p_{\mathrm{MM}} y_{\mathrm{M}}+\lambda_{\mathrm{S}} p_{\mathrm{SM}} y_{\mathrm{S}}\right) x-\left(\lambda_{\mathrm{M}} \alpha_{\mathrm{M}} y_{\mathrm{M}}+\lambda_{\mathrm{S}} \alpha_{\mathrm{S}} y_{\mathrm{S}}\right) y_{\mathrm{M}}-\gamma_{\mathrm{M}} y_{\mathrm{M}} \\
& \frac{d y_{\mathrm{S}}}{d t}=\left(\lambda_{\mathrm{M}} p_{\mathrm{MS}} y_{\mathrm{M}}+\lambda_{\mathrm{S}} p_{\mathrm{SS}} y_{\mathrm{S}}\right) x+\left(\lambda_{\mathrm{M}} \alpha_{\mathrm{M}} y_{\mathrm{M}}+\lambda_{\mathrm{S}} \alpha_{\mathrm{S}} y_{\mathrm{S}}\right) y_{\mathrm{M}}-\gamma_{\mathrm{S}} y_{\mathrm{S}} \\
& \frac{d z_{\mathrm{M}}}{d t}=\gamma_{\mathrm{M}} y_{\mathrm{M}} \\
& \frac{d z_{\mathrm{S}}}{d t}=\gamma_{\mathrm{S}} y_{\mathrm{S}}
\end{aligned}
$$

with initial condition

$$
\boldsymbol{x}(0)=\left(x(0), y_{\mathrm{M}}(0), y_{\mathrm{S}}(0), z_{\mathrm{M}}(0), z_{\mathrm{S}}(0)\right)=\left(1, \mu_{\mathrm{M}}, \mu_{\mathrm{S}}, 0,0\right) .
$$

Further, as described in Section 5.2, a weak convergence result can also be derived using theory for density dependent Markov processes (Ethier and Kurtz [17]) showing that the process $\boldsymbol{V}^{(n)}=\left\{\boldsymbol{V}^{(n)}(t): t \geq 0\right\}$, where

$$
\boldsymbol{V}^{(n)}(t):=\sqrt{n}\left(\overline{\boldsymbol{X}}^{(n)}(t)-\boldsymbol{x}(t)\right) \quad(t \geq 0),
$$

converges to a Gaussian process, whose covariance function can, in principle, be determined. These results can be extended heuristically to hold also for the end of the epidemic, the time of which tends to infinity as $n \rightarrow \infty$, by making a suitable random time transformation. This suggests that the final number of mildly and severely removed cases $\left(Z_{\mathrm{M}}^{(n)}, Z_{\mathrm{S}}^{(n)}\right)$ satisfy a central limit theorem:

$$
\sqrt{n}\left(\begin{array}{c}
\frac{Z_{\mathrm{M}}^{(n)}}{n}-z_{\mathrm{M}}(\infty) \\
Z_{\mathrm{S}}^{(n)} \\
\frac{n}{n}-z_{\mathrm{S}}(\infty)
\end{array}\right) \stackrel{D}{\longrightarrow} N\left(\left(\begin{array}{l}
0 \\
0
\end{array}\right), \Sigma_{Z}(\infty)\right), \quad \text { as } n \rightarrow \infty,
$$

where $\stackrel{D}{\longrightarrow}$ denotes convergence in distribution and $\Sigma_{Z}(\infty)$ is the lower right $2 \times 2$ sub-matrix of the matrix $B \tilde{\Sigma}(\tilde{\tau}) B^{\top}$ in $(5.22)$ and ${ }^{\top}$ denotes transpose. An outline for the heuristic proof 
of this result is given in Section 5.2. The central limit theorem is illustrated in Section 4.2, where simulations of the IEDS model show that the normal approximation works well.

Unfortunately, it is not possible to express the deterministic limit $\left(z_{\mathrm{M}}(\infty), z_{\mathrm{S}}(\infty)\right)$, nor the variance matrix $\Sigma_{Z}(\infty)$ in a very explicit form in order to study their dependence on the model parameters $\left(\lambda_{\mathrm{M}}, \lambda_{\mathrm{S}}, p_{\mathrm{MM}}, p_{\mathrm{MS}}, p_{\mathrm{SM}}, p_{\mathrm{SS}}, \gamma_{\mathrm{M}}, \gamma_{\mathrm{S}}, \alpha_{\mathrm{M}}, \alpha_{\mathrm{S}}\right)$. In Section 4.2 we give some numerical illustrations and also show that the final proportion infected $z_{\mathrm{M}}(\infty)+z_{\mathrm{S}}(\infty)$ may be close to discontinuous in some parameters. Even though the deterministic limits are not very explicit it is possible to get bounds on the final proportion infected, bounds that are more explicit. This is done in the next two subsections.

\subsection{Final outcome of deterministic model}

The first, fourth and fifth equation in (3.6) imply that

$$
\frac{d x}{d t}=-x\left(R_{\mathrm{M}} \frac{d z_{\mathrm{M}}}{d t}+R_{\mathrm{S}} \frac{d z_{\mathrm{S}}}{d t}\right)
$$

where $R_{\mathrm{M}}=\lambda_{\mathrm{M}} / \gamma_{\mathrm{M}}$ and $R_{\mathrm{S}}=\lambda_{\mathrm{S}} / \gamma_{\mathrm{S}}$, which, when integrated, together with the initial condition (3.7), yields

$$
x(t)=\exp \left[-\left(R_{\mathrm{M}} z_{\mathrm{M}}(t)+R_{\mathrm{S}} z_{\mathrm{S}}(t)\right)\right] \quad(t \geq 0) .
$$

Let $\hat{z}_{\mathrm{M}}=z_{\mathrm{M}}(\infty)$ and $\hat{z}_{\mathrm{S}}=z_{\mathrm{S}}(\infty)$ be the final proportion of mild and severe removed cases, respectively. Let $\mu=\mu_{\mathrm{M}}+\mu_{\mathrm{S}}$. Then, letting $t \rightarrow \infty$ in (3.9) and noting that $x(\infty)=$ $1+\mu-\hat{z}_{\mathrm{M}}-\hat{z}_{\mathrm{S}}$, shows that $\left(\hat{z}_{\mathrm{M}}, \hat{z}_{\mathrm{S}}\right)$ satisfies

$$
1+\mu-\hat{z}_{\mathrm{M}}-\hat{z}_{\mathrm{S}}=\exp \left[-\left(R_{\mathrm{M}} \hat{z}_{\mathrm{M}}+R_{\mathrm{S}} \hat{z}_{\mathrm{S}}\right)\right]
$$

If $R_{\mathrm{M}}=R_{\mathrm{S}}$ then it follows from (3.10) that $\hat{z}_{\mathrm{M}}+\hat{z}_{\mathrm{S}}$ satisfies the the usual equation giving the final size of the standard deterministic SIR epidemic, yielding a linear equation satisfied by $\left(\hat{z}_{\mathrm{M}}, \hat{z}_{\mathrm{S}}\right)$. Suppose that $R_{\mathrm{M}} \neq R_{\mathrm{S}}$, and assume without loss of generality that $R_{\mathrm{M}}<R_{\mathrm{S}}$. We describe now some properties of $\left(\hat{z}_{\mathrm{M}}, \hat{z}_{\mathrm{S}}\right)$, formal proofs of which may be found in Appendix A.

Suppose first that $\mu>0$ and let $z_{\mathrm{S}}^{*}$ be the unique positive root of $1+\mu-z=\exp \left(-R_{\mathrm{S}} z\right)$. For $\hat{z}_{\mathrm{S}} \in\left[0, z_{\mathrm{S}}^{*}\right]$, there is a unique $\hat{z}_{\mathrm{M}} \in[0,1+\mu)$ such that $\left(\hat{z}_{\mathrm{M}}, \hat{z}_{\mathrm{S}}\right)$ satisfies (3.10), and there is no solution of (3.10) in the first quadrant with $\hat{z}_{\mathrm{S}}>z_{\mathrm{S}}^{*}$. Thus, the equation (3.10) defines implicitly a function $h:\left[0, z_{\mathrm{S}}^{*}\right] \rightarrow[0,1+\mu)$, where, for $\hat{z}_{\mathrm{S}} \in\left[0, z_{\mathrm{S}}^{*}\right], h\left(\hat{z}_{\mathrm{S}}\right)$ gives the value of $\hat{z}_{\mathrm{M}}$ so that $\left(\hat{z}_{\mathrm{M}}, \hat{z}_{\mathrm{S}}\right)$ satisfies $(3.10)$. The function $h$ is concave and satisfies $h(0)=z_{\mathrm{M}}^{*}$, where $z_{\mathrm{M}}^{*}$ is the unique positive root of $1+\mu-z=\exp \left(-R_{\mathrm{M}} z\right)$, and $h\left(z_{\mathrm{S}}^{*}\right)=0$. The solutions of (3.10) lie on the curve $\left(\hat{z}_{\mathrm{M}}, \hat{z}_{\mathrm{S}}\right)=\left(h\left(\hat{z}_{\mathrm{S}}\right), \hat{z}_{\mathrm{S}}\right)\left(0 \leq \hat{z}_{\mathrm{S}} \leq z_{\mathrm{S}}^{*}\right)$ that joins $\left(z_{\mathrm{M}}^{*}, 0\right)$ to $\left(0, z_{\mathrm{S}}^{*}\right)$ in the first quadrant. Further, if $R_{\mathrm{S}} \geq(1+\mu)^{-1}$ then $h$ is injective if and only if $R_{\mathrm{M}} \geq R_{\mathrm{M}}^{*}$, where $R_{\mathrm{M}}^{*}=\max \left(R_{\mathrm{S}} \log R_{\mathrm{S}} /\left[R_{\mathrm{S}}(1+\mu)-1\right], 0\right)$, while, if $R_{\mathrm{S}}<(1+\mu)^{-1}$ then $h$ is not injective for any 
$R_{\mathrm{M}}<R_{\mathrm{S}}$. (The function $h$ being injective means that whenever $z_{\mathrm{S}}^{(1)}, z_{\mathrm{S}}^{(2)} \in\left[0, z_{\mathrm{S}}^{*}\right]$ are distinct then $h\left(z_{\mathrm{S}}^{(1)}\right) \neq h\left(z_{\mathrm{S}}^{(2)}\right)$.) Moreover, $h\left(\hat{z}_{\mathrm{S}}\right)+\hat{z}_{\mathrm{S}}$ is strictly increasing in $\hat{z}_{\mathrm{S}}$ for $\hat{z}_{\mathrm{S}} \in\left(0, z_{\mathrm{S}}^{*}\right)$. This means that the "proportion" of the population who are ultimately infected by the deterministic IEDS epidemic satisfies $z_{\mathrm{M}}^{*} \leq \hat{z}_{\mathrm{M}}+\hat{z}_{\mathrm{S}} \leq z_{\mathrm{S}}^{*}$. (Recall that it is assumed that $R_{\mathrm{M}}<R_{\mathrm{S}}$. If $R_{\mathrm{M}}>R_{\mathrm{S}}$ then of course the bounds $z_{\mathrm{M}}^{*}$ and $z_{\mathrm{S}}^{*}$ are switched.)

Similar results hold when $\mu=0$. Still assuming that $R_{\mathrm{M}}<R_{\mathrm{S}}$, if $R_{\mathrm{S}} \leq 1$ then $(0,0)$ is the only solution of (3.10) in the first quadrant. If $R_{\mathrm{S}}>1$ then $(0,0)$ is still a solution of (3.10), corresponding to a minor outbreak in the stochastic model, but major outbreaks are now possible and, for large populations, their asymptotic final size satisfies (3.10) with $\left(\hat{z}_{\mathrm{M}}, \hat{z}_{\mathrm{S}}\right) \neq(0,0)$. As in the case $\mu>0$, the equation (3.10) defines implicitly a function $h:\left[0, z_{\mathrm{S}}^{*}\right] \rightarrow[0,1)$, where $z_{\mathrm{S}}^{*}$ is now the non-zero solution of $1-z=\exp \left(-R_{\mathrm{S}} z\right)$. All the above results concerning the function $h$ and the behaviour of $h\left(\hat{z}_{\mathrm{S}}\right)+\hat{z}_{\mathrm{S}}$ continue to hold, except now $z_{\mathrm{M}}^{*}$ is given by the largest real solution of $1-z=\exp \left(-R_{\mathrm{M}} z\right)$. Hence, $z_{\mathrm{M}}^{*}=0$ if $R_{\mathrm{M}} \leq 1$ and $z_{\mathrm{M}}^{*}>0$ if $R_{\mathrm{M}}>1$. Thus, if $R_{\mathrm{M}} \leq 1$, the solutions of (3.10) lie on a curve that joins $(0,0)$ to $\left(0, z_{\mathrm{S}}^{*}\right)$, whilst, if $R_{\mathrm{M}}>1$, they lie on a curve that joins $\left(z_{\mathrm{M}}^{*}, 0\right)$ to $\left(0, z_{\mathrm{S}}^{*}\right)$, where $z_{\mathrm{M}}^{*}>0$.

Note that in general (3.10) does not determine the final outcome of the deterministic IEDS epidemic. Except for a few very special cases, discussed in Ball and Britton [4], Section 2.2.2, it is not possible to derive a second, independent equation satisfied by $\left(\hat{z}_{\mathrm{M}}, \hat{z}_{\mathrm{S}}\right)$.

\subsection{Bounds on the final size}

Let $\underline{R}_{0}=\min \left(R_{\mathrm{M}}, R_{\mathrm{S}}\right)$ and $\bar{R}_{0}=\max \left(R_{\mathrm{M}}, R_{\mathrm{S}}\right)$. Then the theory in Section 3.3 shows that, for $\mu_{\mathrm{M}} \geq 0$ and $\mu_{\mathrm{S}} \geq 0$, the final fraction infected in the deterministic IEDS model, $z_{\mathrm{M}}(\infty)+z_{\mathrm{S}}(\infty)$, satisfies

$$
z_{\min } \leq z_{\mathrm{M}}(\infty)+z_{\mathrm{S}}(\infty) \leq z_{\max }
$$

where $z_{\min }$ and $z_{\max }$ are the largest roots of

$$
1+\mu_{\mathrm{M}}+\mu_{\mathrm{S}}-z=\mathrm{e}^{-\underline{R}_{0} z} \text { and } 1+\mu_{\mathrm{M}}+\mu_{\mathrm{S}}-z=\mathrm{e}^{-\bar{R}_{0} z},
$$

respectively. Thus the limiting proportion ever infected in the deterministic IEDS model is bounded between those of the general deterministic epidemic with basic reproduction numbers $\underline{R}_{0}$ and $\bar{R}_{0}$. The smaller the difference between $\underline{R}_{0}$ and $\bar{R}_{0}$, the narrower the bounds $(3.11)$ become. If $\underline{R}_{0}=\bar{R}_{0}$, then $z_{\text {min }}=z_{\text {max }}$ and the model is closely related to the general epidemic model, except that some infectives are labelled mild and others severe.

Suppose that $\mu_{\mathrm{M}}=\mu_{\mathrm{S}}=0$. If $p_{\mathrm{MM}}+p_{\mathrm{SM}} \rightarrow 0$ and/or $\alpha_{\mathrm{M}}+\alpha_{\mathrm{S}} \rightarrow \infty$ (implying that nearly all infectives are severe cases), then $z_{\mathrm{M}}(\infty) \rightarrow 0$ and $z_{\mathrm{S}}(\infty)$ converges to $z_{\max }$ if $\bar{R}_{0}=R_{\mathrm{S}}$ (the typical case) and to $z_{\text {min }}$ otherwise. Similarly, if $p_{\mathrm{MS}}+p_{\mathrm{SS}} \rightarrow 0$ and $\alpha_{\mathrm{M}}+\alpha_{\mathrm{S}} \rightarrow 0$ (so nearly all infectives are mild), then $z_{\mathrm{S}}(\infty) \rightarrow 0$ and $z_{\mathrm{M}}(\infty)$ converges to $z_{\min }$ if $\underline{R}_{0}=R_{\mathrm{M}}$ (the typical case) and to $z_{\max }$ otherwise. Essentially the same results hold if $\mu_{\mathrm{M}}+\mu_{\mathrm{S}}>0$. 
Similar bounds to (3.11) hold for the stochastic IEDS model. Specifically,

$$
Z_{\min } \stackrel{s t}{\leq} Z_{\mathrm{M}}(\infty)+Z_{\mathrm{S}}(\infty) \stackrel{s t}{\leq} Z_{\max }
$$

where $Z_{\min }$ and $Z_{\max }$ are the total sizes of the general stochastic epidemics, with initially $m_{\mathrm{M}}+m_{\mathrm{S}}$ infectives and $n$ susceptibles, having basic reproduction numbers $\underline{R}_{0}$ and $\bar{R}_{0}$, respectively, and $\stackrel{s t}{\leq}$ denotes stochastically less than. The stochastic inequalities (3.12) are proved in Appendix B by extending the construction of Sellke [18] to the IEDS model.

\subsection{Effects of vaccination}

We now analyse the effect of vaccinating a proportion $v$ of susceptibles prior to an outbreak.

\subsubsection{The all-or-nothing vaccine response}

We start with the all-or-nothing response, where a vaccinated individual is completely immune with probability $e$ (for efficacy) and the vaccination has no effect with the remaining probability $1-e$. It is easy to model the effects of this vaccine response. In a large community, a fraction $1-v+v(1-e)=1-v e$ are (fully) susceptible and the remaining fraction $v e$ are completely immune, and that is all that is changed compared to the situation without vaccination.

This implies that we can approximate the initial stages with a two-type branching process having the same death rates as before but with all birth rates reduced by a factor ve, so the former birth rates should all be multiplied by the factor $1-v e$. The matrix of mean offspring is hence the former (equation 3.1) multiplied by $1-v e$, and the new reproduction number equals the former $\left(R_{0}\right.$ defined in (3.2)) multiplied by $1-v e$ :

$$
R_{v}^{(A o N)}=(1-v e) R_{0}
$$

The critical vaccination coverage $v_{c}^{(A o N)}$ is the fraction $v$ necessary to reduce $R_{v}^{(A o N)}$ down to 1. From the form of $R_{v}^{(A o N)}$, it is immediately seen that, when $1<R_{0} \leq(1-e)^{-1}$, the critical vaccination coverage is given by

$$
v_{c}^{(A o N)}=\frac{1}{e}\left(1-\frac{1}{R_{0}}\right)
$$

If $R_{0}>(1-e)^{-1}$, vaccination alone cannot be sure of preventing a major outbreak.

Starting with few initial infectives, $R_{v}^{(A o N)} \leq 1$ implies that there can be no major outbreak whereas if $R_{v}^{(A o N)}>1$ there is a possibility for a major outbreak. The probability for a major outbreak may also be derived using branching process theory, similar to the case without vaccination. If the epidemic does take off the epidemic process may be approximated by 
the same set of differential equations as without vaccination, equation (3.6), but with initial condition $\left(x(0), y_{\mathrm{M}}(0), y_{\mathrm{S}}(0), z_{\mathrm{M}}(0), z_{\mathrm{S}}(0)\right)=\left(1-v e, \mu_{\mathrm{M}}, \mu_{\mathrm{S}}, 0,0\right)$. In Section 4.3 we illustrate how the limiting final size of the two types change with $v$ for fixed vaccine efficacy $e$ and IEDS model parameters.

\subsubsection{The non-random vaccine response}

For the non-random vaccine response things are a bit more complicated. As before it is possible to approximate the initial stages by a branching process to derive a reproduction number $R_{v}^{(N R)}$ and to approximate the epidemic process by a deterministic system in the event that the epidemic takes off. The difference is that we now have four types of infectious individual: mild unvaccinated, severe unvaccinated, mild vaccinated and severe vaccinated. The mean offspring matrix for the approximating branching process is hence a $4 \times 4$ matrix. However, its form is quite similar to the offspring matrix without vaccination. For instance, the expected number of mild unvaccinated that a mild unvaccinated infects during its infectious period equals $\mu_{\mathrm{MU}, \mathrm{MU}}=\left(\lambda_{\mathrm{M}} / \gamma_{\mathrm{M}}\right) p_{\mathrm{MM}}(1-v)=(1-v) \mu_{\mathrm{MM}}$ and the average number of mild vaccinated individuals this person infects equals $\mu_{\mathrm{MU}, \mathrm{MV}}=\left(\lambda_{\mathrm{M}} / \gamma_{\mathrm{M}}\right) p_{\mathrm{MM}} v a=v a \mu_{\mathrm{MM}}$. This is true because the fraction $v$ are vaccinated, and each vaccinated individual has relative risk $a$ of becoming infected, compared to an unvaccinated individual. Similarly, a mild vaccinated infects, on average, $\mu_{\mathrm{MV}, \mathrm{MU}}=b^{\theta}\left(\lambda_{\mathrm{M}} / b^{\theta-1} \gamma_{\mathrm{M}}\right) p_{\mathrm{MM}}(1-v)=b\left(\lambda_{\mathrm{M}} / \gamma_{\mathrm{M}}\right) p_{\mathrm{MM}}(1-v)=(1-v) b \mu_{\mathrm{MM}}$ mild unvaccinated and $\mu_{\mathrm{MV}, \mathrm{MV}}=b\left(\lambda_{\mathrm{M}} / \gamma_{\mathrm{M}}\right) p_{\mathrm{MM}} v a=v a b \mu_{\mathrm{MM}}$ mild vaccinated. The other terms in the mean offspring distributions are obtained similarly. It is worth noting that the parameter $\theta$, quantifying if the reduced infection pressure of vaccinees comes from lower infectivity and/or shorter infectious period, does not enter the mean offspring matrix. Consequently, this parameter does not have an effect on determining whether or not an outbreak may occur - however, in Section 4.3 we see that it does have an effect on how many are infected if an outbreak actually occurs.

Let $M_{v}^{(N R)}$ denote the mean offspring matrix of the above branching process, with the types ordered MU, SU, MV, SV. Then,

$$
M_{v}^{(N R)}\left[\begin{array}{llll}
(1-v) \mu_{\mathrm{MM}} & (1-v) \mu_{\mathrm{MS}} & v a \mu_{\mathrm{MM}} & v a \mu_{\mathrm{MS}} \\
(1-v) \mu_{\mathrm{SM}} & (1-v) \mu_{\mathrm{SS}} & v a \mu_{\mathrm{SM}} & v a \mu_{\mathrm{SS}} \\
(1-v) b \mu_{\mathrm{MM}} & (1-v) b \mu_{\mathrm{MS}} & v a b \mu_{\mathrm{MM}} & v a b \mu_{\mathrm{MS}} \\
(1-v) b \mu_{\mathrm{SM}} & (1-v) b \mu_{\mathrm{SS}} & v a b \mu_{\mathrm{SM}} & v a b \mu_{\mathrm{SS}}
\end{array}\right]=V_{a b}^{v} \otimes M,
$$

where $\otimes$ denotes Kronecker product and

$$
V_{a b}^{v}=\left[\begin{array}{ll}
1-v & v a \\
(1-v) b & v a b
\end{array}\right]
$$

The reproduction number $R_{v}^{(N R)}$ is given by the largest eigenvalue $M_{v}^{(N R)}$, which, in view of (3.15) is the product of the largest eigenvalues of $V_{a b}^{v}$ and $M$. Recall that the largest eigenvalue 
of $M$ is $R_{0}$. The matrix $V_{a b}^{v}$ has rank one, so its largest eigenvalue equals its trace, $1-v(1-a b)$, whence

$$
R_{v}^{(N R)}=(1-v(1-a b)) R_{0}
$$

We see that this is very similar to the reproduction number $R_{v}^{(A o N)}$ in (3.13), the only difference being that $e$ is replaced by $1-a b$. The factor $1-a b$ may be interpreted as the vaccine efficacy for the non-random vaccine response, as noted in Section 2.2. The critical vaccination coverage $v_{c}^{(N R)}$ hence has the following form, assuming $1<R_{0} \leq(a b)^{-1}$ :

$$
v_{c}^{(N R)}=\frac{1}{1-a b}\left(1-\frac{1}{R_{0}}\right) .
$$

When $R_{0}>1 /(a b)$, then vaccination alone cannot surely prevent a major outbreak occurring. If $R_{v}^{(N R)}>1$ the epidemic may take off even if the epidemic is initiated by few infectives. If the community size $n$ is large the epidemic may then be approximated by the following set of differential equations:

$$
\begin{aligned}
\frac{d x_{\mathrm{U}}}{d t} & =-\left(\lambda_{\mathrm{M}}\left(y_{\mathrm{MU}}+b^{\theta} y_{\mathrm{MV}}\right)+\lambda_{\mathrm{S}}\left(y_{\mathrm{SU}}+b^{\theta} y_{\mathrm{SV}}\right)\right) x_{\mathrm{U}} \\
\frac{d x_{\mathrm{V}}}{d t} & =-a\left(\lambda_{\mathrm{M}}\left(y_{\mathrm{MU}}+b^{\theta} y_{\mathrm{MV}}\right)+\lambda_{\mathrm{S}}\left(y_{\mathrm{SU}}+b^{\theta} y_{\mathrm{SV}}\right)\right) x_{\mathrm{V}} \\
\frac{d y_{\mathrm{MU}}}{d t} & =\left(\lambda_{\mathrm{M}} p_{\mathrm{MM}}\left(y_{\mathrm{MU}}+b^{\theta} y_{\mathrm{MV}}\right)+\lambda_{\mathrm{S}} p_{\mathrm{SM}}\left(y_{\mathrm{SU}}+b^{\theta} y_{\mathrm{SV}}\right)\right) x_{\mathrm{U}}-\left(\lambda_{\mathrm{M}} \alpha_{\mathrm{M}} y_{\mathrm{M}}+\lambda_{\mathrm{S}} \alpha_{\mathrm{S}} y_{\mathrm{S}}\right) y_{\mathrm{MU}}-\gamma_{\mathrm{M}} y_{\mathrm{MU}} \\
\frac{d y_{\mathrm{MV}}}{d t} & =a\left(\lambda_{\mathrm{M}} p_{\mathrm{MM}}\left(y_{\mathrm{MU}}+b^{\theta} y_{\mathrm{MV}}\right)+\lambda_{\mathrm{S}} p_{\mathrm{SM}}\left(y_{\mathrm{SU}}+b^{\theta} y_{\mathrm{SV}}\right)\right) x_{\mathrm{V}}-\left(\lambda_{\mathrm{M}} \alpha_{\mathrm{M}} y_{\mathrm{M}}+\lambda_{\mathrm{S}} \alpha_{\mathrm{S}} y_{\mathrm{S}}\right) y_{\mathrm{MV}}-b^{\theta-1} \gamma_{\mathrm{M}} y_{\mathrm{MV}} \\
\frac{d y_{\mathrm{SU}}}{d t} & =\left(\lambda_{\mathrm{M}} p_{\mathrm{MS}}\left(y_{\mathrm{MU}}+b^{\theta} y_{\mathrm{MV}}\right)+\lambda_{\mathrm{S}} p_{\mathrm{SS}}\left(y_{\mathrm{SU}}+b^{\theta} y_{\mathrm{SV}}\right)\right) x_{\mathrm{U}}+\left(\lambda_{\mathrm{M}} \alpha_{\mathrm{M}} y_{\mathrm{M}}+\lambda_{\mathrm{S}} \alpha_{\mathrm{S}} y_{\mathrm{S}}\right) y_{\mathrm{MU}}-\gamma_{\mathrm{S}} y_{\mathrm{SU}} \\
\frac{d y_{\mathrm{SV}}}{d t} & =a\left(\lambda_{\mathrm{M}} p_{\mathrm{MS}}\left(y_{\mathrm{MU}}+b^{\theta} y_{\mathrm{MV}}\right)+\lambda_{\mathrm{S}} p_{\mathrm{SS}}\left(y_{\mathrm{SU}}+b^{\theta} y_{\mathrm{SV}}\right)\right) x_{\mathrm{V}}+\left(\lambda_{\mathrm{M}} \alpha_{\mathrm{M}} y_{\mathrm{M}}+\lambda_{\mathrm{S}} \alpha_{\mathrm{S}} y_{\mathrm{S}}\right) y_{\mathrm{MV}}-b^{\theta-1} \gamma_{\mathrm{S}} y_{\mathrm{SV}} \\
\frac{d z_{\mathrm{MU}}}{d t} & =\gamma_{\mathrm{M}} y_{\mathrm{MU}} \\
\frac{d z_{\mathrm{MV}}}{d t} & =b^{\theta-1} \gamma_{\mathrm{M}} y_{\mathrm{MV}} \\
\frac{d z_{\mathrm{SU}}}{d t} & =\gamma_{\mathrm{S}} y_{\mathrm{SU}} \\
\frac{d z_{\mathrm{SV}}}{d t} & =b^{\theta-1} \gamma_{\mathrm{S}} y_{\mathrm{SV}}
\end{aligned}
$$

with initial condition $x_{\mathrm{U}}(0)=1-v, x_{\mathrm{V}}(0)=v, y_{\mathrm{MU}}(0)=\mu_{\mathrm{M}}, y_{\mathrm{SU}}(0)=\mu_{\mathrm{S}}$, and the remaining coordinates equal to 0 .

By analysing this system of differential equations it is possible to see what fraction of the different types are removed at the end of the epidemic, i.e. to study $z_{\mathrm{MU}}(\infty), z_{\mathrm{MV}}(\infty), z_{\mathrm{MV}}(\infty)$ and $z_{\mathrm{MV}}(\infty)$. In Section 4.3 we give some numerical illustrations which show how the proportion ultimately infected varies with the vaccine coverage $v$. It is also seen there that these proportions depend on the vaccine response parameter $\theta$. 


\section{$4 \quad$ Numerical examples}

Below we give some examples supporting our limiting results and illustrating some interesting features of the model.

\subsection{Illustration of branching process approximation}

In Section 3.1 it is explained that the distribution of the final number infected of the two types, $\left(Z_{\mathrm{M}}^{(n)}, Z_{\mathrm{S}}^{(n)}\right)$, converges to the distribution of $\left(Z_{\mathrm{M}}, Z_{\mathrm{S}}\right)$, the total numbers ever born in a certain two-type branching process. In particular, the final numbers of infected tend to infinity (with $n$ ) with the same probability as the branching process grows beyond all limits. This means that for finite but fairly large $n$, the distribution of $\left(Z_{\mathrm{M}}^{(n)}, Z_{\mathrm{S}}^{(n)}\right)$ is concentrated on two parts: small numbers (minor outbreak) or close to some large deterministic value (obtained from the deterministic model) of order $n$. This is illustrated in Figure 1 where we show histograms of the final number infected (of either type) from 10000 simulations for a specific set of parameter values, namely $\gamma_{\mathrm{M}}=\gamma_{\mathrm{S}}=1, \lambda_{\mathrm{M}}=1 \lambda_{\mathrm{S}}=2.5, \alpha_{\mathrm{M}}=\alpha_{\mathrm{S}}=1$, $p_{\mathrm{MM}}=0.8, p_{\mathrm{MS}}=0.2, p_{\mathrm{SM}}=0.2$, and $p_{\mathrm{SS}}=0.8$ and different community sizes. Thus both mild and severe infectives are assumed to infect new individuals to the same type as themselves with probability 0.8 and, using $(3.2), R_{0}=2.0782$. Each epidemic was initiated with one mild and one severe infective, so $m_{\mathrm{M}}=m_{\mathrm{S}}=1$. It is seen that the distribution is bimodal already for $n=100$ and when $n \geq 500$ there is a large region between the two modes having no empirical support. Consequently, the notion of minor and major outbreak makes sense once the population size is moderate (e.g. 500) or larger. For smaller community sizes the distinction between minor and major outbreaks is not as clear.

Our next illustration concerns the determination of the probability of a major outbreak approximated from branching process theory (Section 3.1). To illustrate this we performed simulations of the epidemic in communities of various sizes. In the simulations all the parameter values except $\alpha_{\mathrm{M}}$ and $\alpha_{\mathrm{S}}$ were the same as above. Since the approximating branching process has no switching from mild to severe one would expect the approximation to be better the less frequent such switches are in the epidemic, i.e. the smaller $\alpha_{\mathrm{M}}$ and $\alpha_{\mathrm{S}}$ are. For this reason simulations were performed for three situations, all having $\alpha_{\mathrm{M}}=\alpha_{\mathrm{S}}=$ : $\alpha$. The three situations were $\alpha=0,1$ and 10. Starting with one mild and one severe infected, 10000 simulations were performed, for a range of different community sizes. In Table 1 we list the fraction of simulations that resulted in a major outbreak. A "major outbreak" was defined as an outbreak resulting in more than $30 \%$ infected (of either type) - as seen in Figure 1 the results are not very sensitive to the particular choice of boundary between minor and major outbreaks.

Using the results of Section 3.1, we computed the theoretical probability for the branching process growing beyond all limits, which approximates the probability of a major outbreak for the IEDS model. Starting with one mild and one severe infective we obtained that 

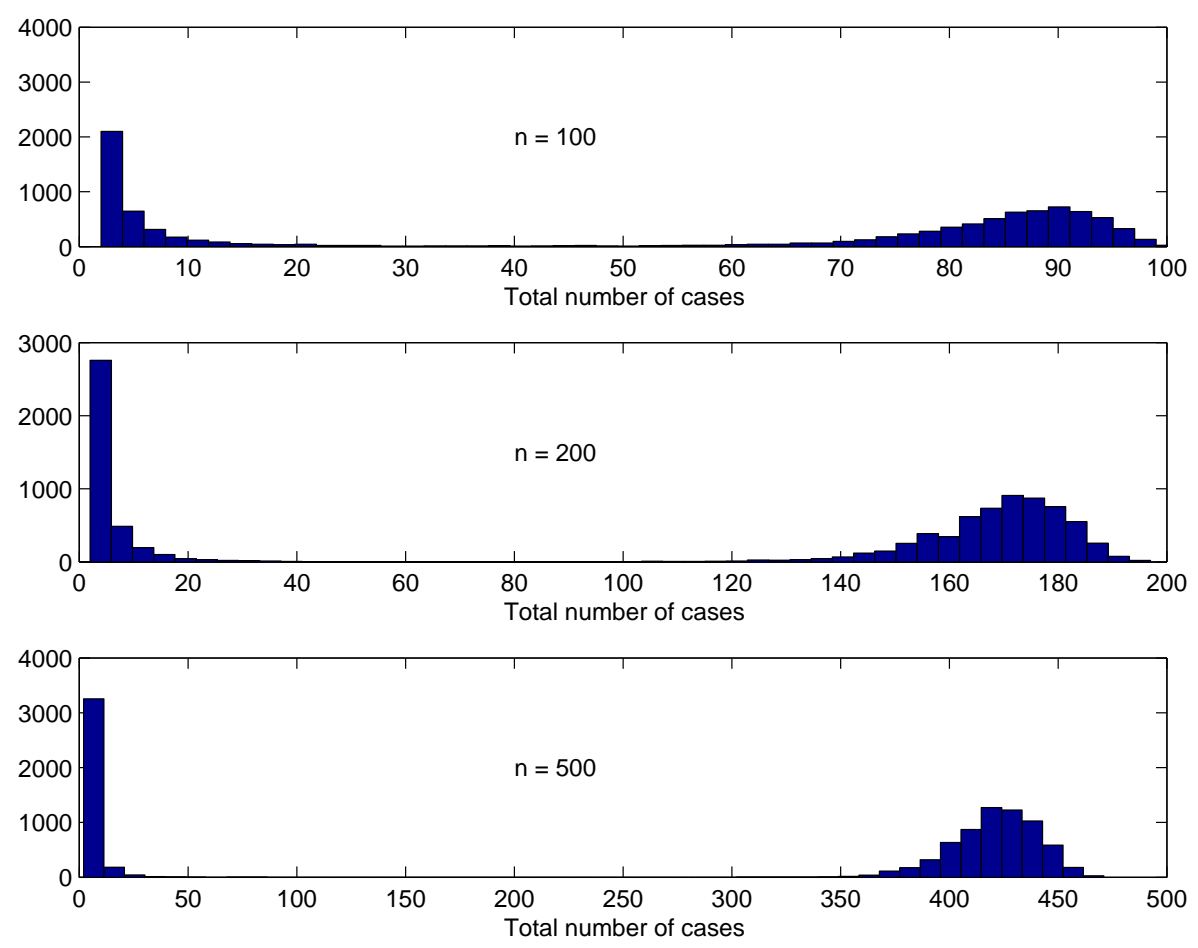

Figure 1: Histograms of final number removed (of either type) from 10000 simulations of the IEDS model for a few different community sizes. See text for more details.

$P\left(\right.$ major outbreak $\left.\mid m_{\mathrm{M}}=1, m_{\mathrm{S}}=1\right)=0.6479$. The approximating branching process has no switches between mild and severe, so this outbreak probability approximates all epidemics, irrespective of $\alpha$ (and $n$ ). From the table it is seen that the theoretical outbreak probability agrees well with the empirical fractions once the population size is 500 or larger, and this holds for all choices of $\alpha$. Of course, there is some randomness from using 10000 simulations. The standard deviation for the empirical fractions is approximately 0.0048 , so a $95 \%$ confidence interval for the theoretical outbreak probability would approximately be the observed fraction plus or minus 0.01 . Hence a hypothesis test would not reject, at the $5 \%$ level, 0.6479 as the theoretical outbreak probability for any set of simulations with $n=500$ or larger.

Observe from Table 1 that, for small $n$, the empirical probability of a major outbreak generally increases with $\alpha$. This is because $R_{\mathrm{S}}>R_{\mathrm{M}}$ (see Section 3.3), so reinfection of a mild infective increases the chance that an outbreak becomes established. This effect becomes less marked as $n$ increases, since the chance of reinfection in the early stages of an outbreak decreases, and is absent in the limit $n \rightarrow \infty$. 
Table 1: Fraction of major outbreaks in 10000 simulations of the IEDS model for different community sizes $n$ and different $\alpha$. See text for further details.

\begin{tabular}{c|ccc}
\hline$n$ & $\alpha=0$ & $\alpha=1$ & $\alpha=10$ \\
\hline 100 & 0.6137 & 0.6316 & 0.6729 \\
200 & 0.6320 & 0.6316 & 0.6703 \\
500 & 0.6399 & 0.6501 & 0.6565 \\
1000 & 0.6489 & 0.6436 & 0.6539 \\
5000 & 0.6450 & 0.6430 & 0.6455 \\
10000 & 0.6495 & 0.6505 & 0.6489 \\
100000 & 0.6511 & 0.6490 & 0.6511 \\
\hline
\end{tabular}

\subsection{Illustration of central limit theorem and final size}

Having investigated the branching process approximation we now focus on major outbreaks by having $R_{0}>1$ and starting with a fair number of infectives. For this situation we performed simulations to assess how well the central limit approximation works for finite populations. Figure 2 is based on 10000 simulations of the IEDS model for a population consisting of $n=100000$ individuals, with the epidemic parameters being the same as those used in Figure 1. Recall that $R_{0}=2.0782$, so the epidemic is above threshold. To avoid minor outbreaks, each simulation was initiated by 100 mild and 100 severely infectious individuals. The figure contains histograms of the final number of mild (left) and severe (right) cases from the simulations. The average scaled (i.e. divided by the initial number of susceptibles, $n$ ) numbers of mildly and severely infected in the simulations, including initial infectives, were 0.1776 and 0.6659 , respectively. The corresponding theoretical values, obtained using methods described in Section 3.3, were $z_{\mathrm{M}}(\infty)=0.1776$ and $z_{\mathrm{S}}(\infty)=0.6660$, i.e. essentially the same as the empirical means. The limiting covariance matrix $\Sigma_{Z}(\infty)$ (see equation (3.8)) was computed numerically. The limiting scaled variances and covariance (now multiplied by $n$ ) for the proportions infected (elements $(1,1),(2,2)$ and $(1,2)$ of $\Sigma_{Z}(\infty)$ in equation $(3.8)$ ) were $\sigma_{11}(\infty)=0.3184, \sigma_{22}(\infty)=1.2297$ and $\sigma_{12}(\infty)=-0.4248$. The corresponding empirical variances from the simulations were $0.3161,1.2076$ and -0.4135 , respectively, again close to their asymptotic counterparts. In Figure 2, the probability density functions of the approximating normal distributions, with means $n z_{\mathrm{M}}(\infty)$ and $n z_{\mathrm{S}}(\infty)$, and variances $n \sigma_{11}(\infty)$ and $n \sigma_{22}(\infty)$, are superimposed. (The probability density functions are scaled so that the areas under them match those of the histograms.) As can be seen, there is excellent agreement between the empirical distributions and the theoretical asymptotic normal distributions.

We now illustrate that the final size of the limiting deterministic model can be close to discontinuous in certain model parameters. In Figure 3 we consider epidemics with parameters $\gamma_{\mathrm{M}}=1, \gamma_{\mathrm{S}}=1, \lambda_{\mathrm{M}}=0.8, \lambda_{\mathrm{S}}=5, p_{\mathrm{MM}}=1, p_{\mathrm{MS}}=0, p_{\mathrm{SM}}=1, p_{\mathrm{SS}}=0$, and initial proportions infected $\mu_{\mathrm{M}}=0.01$ and $\mu_{\mathrm{S}}=0$. The reinfection parameters are varied by setting $\alpha_{\mathrm{M}}=\alpha_{\mathrm{S}}=\alpha$ 

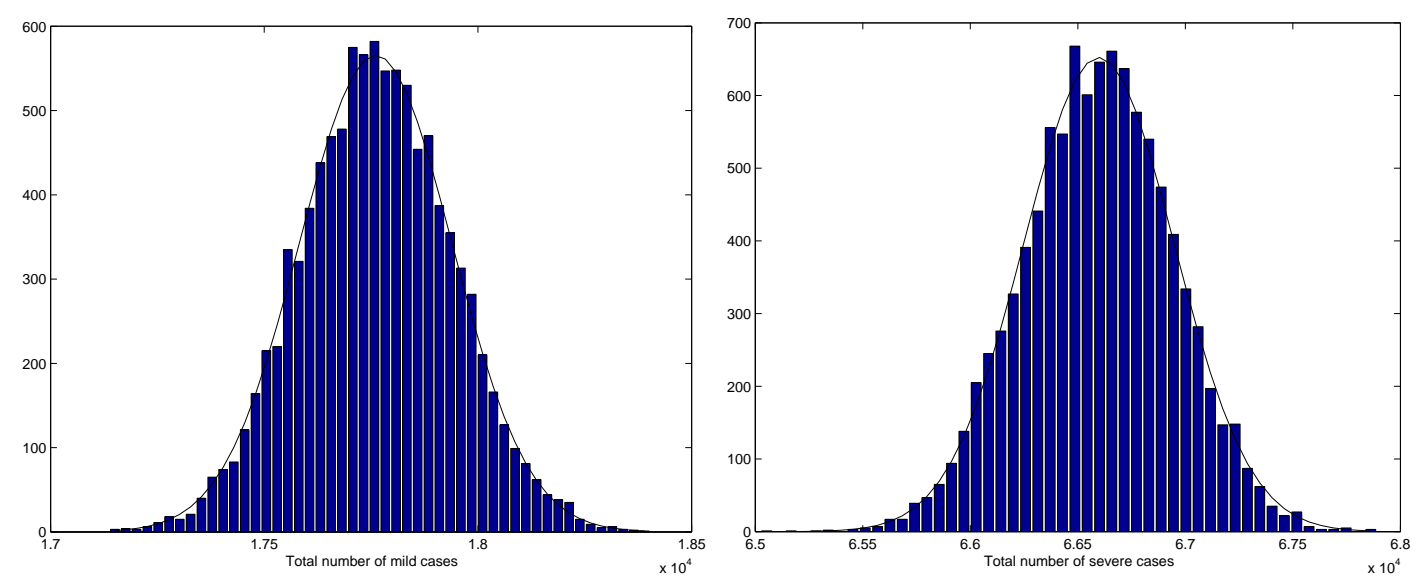

Figure 2: Histograms of final outcome from 10000 simulations of the IEDS model in a community of 100000 individuals, with theoretical normal approximation superimposed. To the left are the number of mildly infected and to the right the number of severely infected.

and letting $\alpha$ vary from 0 to 10 . The figure shows the total fraction ultimately infected, $z_{\mathrm{M}}(\infty)+z_{\mathrm{S}}(\infty)$ as a function of $\alpha$. We see that $z_{\mathrm{M}}(\infty)+z_{\mathrm{S}}(\infty)$ is close to discontinuous in $\alpha$ when $\alpha \approx 7.43$. A similar phenomenon (but with an actual discontinuity) was observed in the EDS model (Ball and Britton [9]) and in some deterministic models for endemic diseases (e.g. Greenhalgh et al. [19] and van den Driessche and Watmough [20]). A possible explanation for this phenomenon in the present model is as follows. Note that the model is below threshold, as $R_{0}\left(=R_{\mathrm{M}}\right)=0.8<1$. However, the initial proportion mildly infected $\mu_{\mathrm{M}}>0$ makes it less subcritical, since mild infectives can be reinfected to become severe infectives, whose infection parameters are well above threshold $\left(R_{\mathrm{S}}=5\right)$. As $\alpha$ increases, there is more reinfection and hence more severe infectives, which leads to even more reinfection since severe infectives reinfect at an appreciably higher rate than mild infectives. Thus there is positive feedback, leading to a steep rise in the fraction ultimately infected as $\alpha$ passes a certain value.

\subsection{Final size as function of $v$}

We now illustrate what effect the vaccine response models have on the fractions of ultimately mild and severely infected in the limiting deterministic epidemic processes.

First we compare the final outcome using an all-or-nothing vaccine and a leaky vaccine, both having vaccine efficacy $e=0.75$, as a function of the fraction vaccinated $v$. The model parameters are chosen to be $\lambda_{\mathrm{M}}=1, \lambda_{\mathrm{S}}=4, \gamma_{\mathrm{M}}=1, \gamma_{\mathrm{S}}=1, p_{\mathrm{MM}}=0.8, p_{\mathrm{MS}}=0.2$, $p_{\mathrm{SM}}=$ $0.2, p_{\mathrm{SS}}=0.8, \alpha_{\mathrm{M}}=1, \alpha_{\mathrm{S}}=1$, with initial fractions of mild and severe infectives given by $\mu_{\mathrm{M}}=10^{-6}$ and $\mu_{\mathrm{S}}=0$, respectively. Thus the epidemic parameters are the same as those used in Figure 1, except that $\lambda_{\mathrm{S}}$ is increased to 4 to highlight better the effect of vaccination. Figure 4 shows the final fractions infected of the two types for these parameter values and 


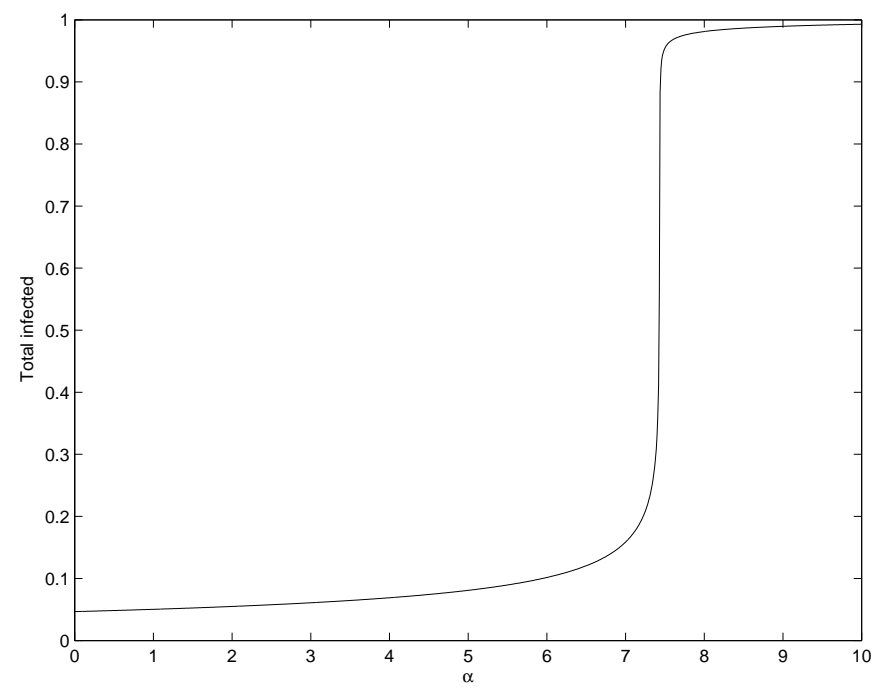

Figure 3: Total fraction ultimately infected (of either type) as a function of $\alpha$. See text for further details.

varying the vaccination coverage $v$.

It is seen that, for these particular parameter values, vaccination has greatest impact on the number of severely infected. It is also observed that the all-or-nothing vaccine outperforms the leaky vaccine having the same efficacy. This observation is true in general and has been explained by the fact that the first contact with a vaccinee under either type of vaccine response has the same risk of resulting in infection, whereas additional contacts (with not yet infected!) never result in infection under the all-or-nothing vaccine response but may result infection under the leaky vaccine response (Ball and Becker [21]). Finally, even though the all-or-nothing vaccine response is better in this sense, both responses have the same critical vaccination coverage $v_{c}=0.9250\left(R_{0}=3.2649\right.$ from $\left.(3.2)\right)$, as is seen by setting $(a, b)=(1-e, 1)$ in $(3.18)$ and comparing with (3.14).

Note from Figure 4 that, under the leaky vaccine, the fraction mildly infected initially increases slightly with the vaccination coverage. This effect is more marked for other parameter values. Figure 5 plots the final fractions infected for the all-or-nothing and leaky vaccines, both having efficacy $e=0.75$, and model parameters set to $\lambda_{\mathrm{M}}=2, \lambda_{\mathrm{S}}=5, \gamma_{\mathrm{M}}=1, \gamma_{\mathrm{S}}=1, p_{\mathrm{MM}}=$ $1, p_{\mathrm{MS}}=0, p_{\mathrm{SM}}=1, p_{\mathrm{SS}}=0, \alpha_{\mathrm{M}}=10, \alpha_{\mathrm{S}}=10, \mu_{\mathrm{M}}=10^{-6}, \mu_{\mathrm{S}}=0$. As before, the all-ornothing vaccine response outperforms the leaky vaccine response having the same efficacy. We note also that the fraction mildly infected initially increases appreciably with the vaccination coverage for both vaccine responses. The explanation to this is that even though the overall fraction infected decreases, the proportion severely infected decreases much faster since fewer and fewer switch from mild to severe, thus making the proportion of mildly infected increase. Our final comparison concerns the non-random vaccine response model, and in particular 


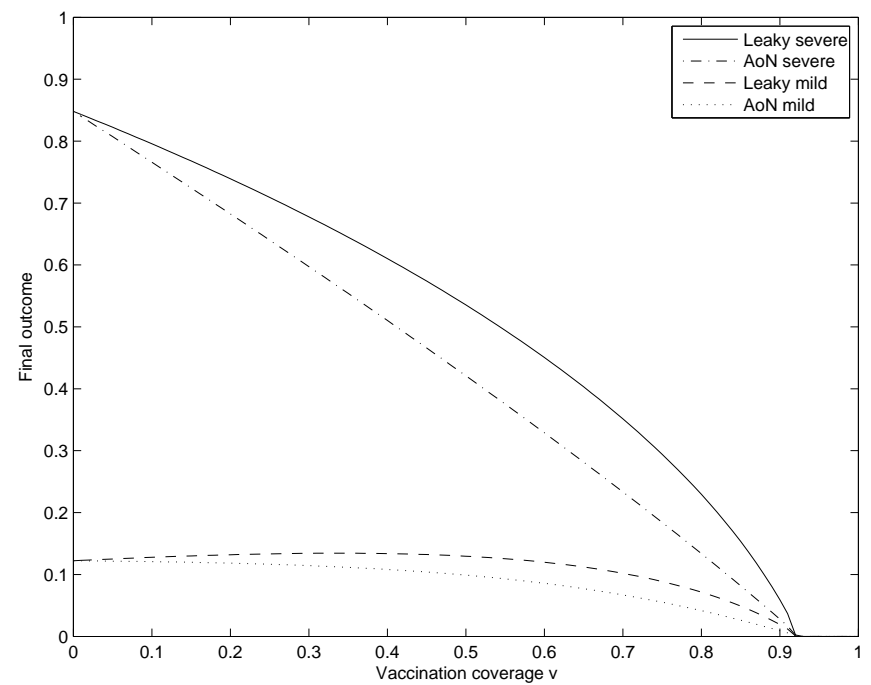

Figure 4: Final fractions infected (of the two types) as a function of the vaccination coverage $v$, under the all-or-nothing and leaky vaccine models.

compares the effect of reducing infectivity $(\theta=1)$ with the effect of reducing the length of the infectious period $(\theta=0)$. In Figure 6 we plot the fractions ultimately infected of the two types for both vaccine responses, as a function of the vaccination coverage. The epidemic model parameters are the same as in Figure 5 , and the vaccine parameters are set to $a=b=0.5$ in the non-random vaccine response model, resulting in efficacy $1-a b=0.75$ as before. It is seen that the two vaccines perform differently although the difference is only moderate. There are fewer severely infected and more mildly infected when the infectious period is reduced and not the infectivity $(\theta=0)$ compared to the opposite $(\theta=1)$. A possible explanation for this is that reducing the infectious period mainly implies fewer switches from mild to severe, whereas as reducing infectivity brings down the infectious pressure in general. In an equivalent figure (not shown), where the epidemic parameters are the same as in Figure 4, the curves for the two vaccine responses $(\theta=0$ and $\theta=1)$ are virtually identical. Note that with these latter epidemic parameters, individuals may become severe cases when first infected and there is less reinfection.

\section{Proofs}

\subsection{Branching process approximation}

The branching process approximation of the initial stages of the IEDS epidemic may be made fully rigorous by extending the coupling argument of Ball and Donnelly [22]. We consider a sequence of IEDS epidemics, $\left\{E^{(n)}: n \geq 1\right\}$ say, with the epidemic $E^{(n)}$ having initially 


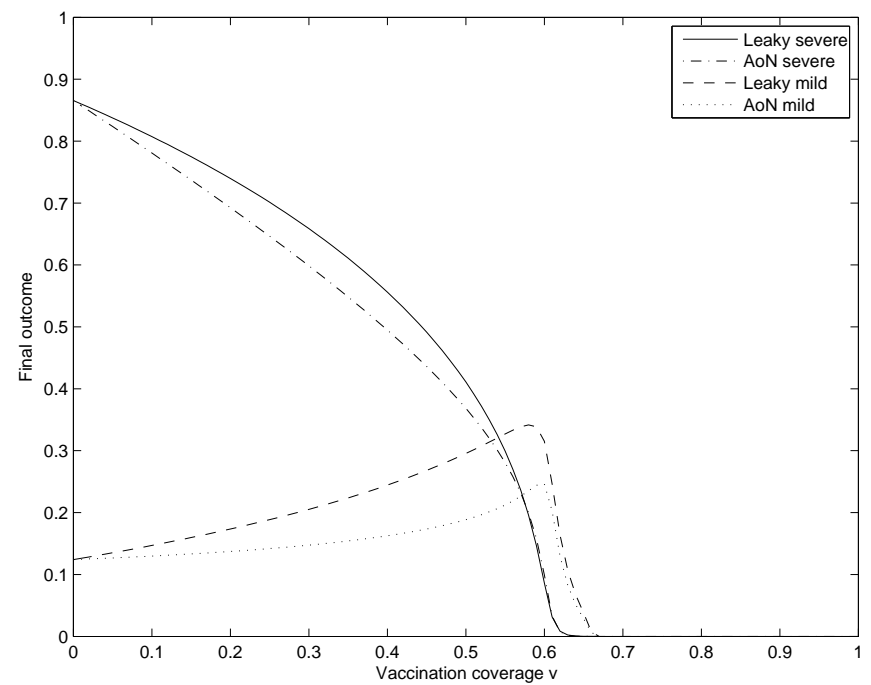

Figure 5: Final fractions infected as a function of the vaccination coverage under the all-ornothing and leaky vaccine models.

(i.e. at time $t=0) n$ susceptibles, $m_{\mathrm{M}}$ mild infectives, $m_{\mathrm{S}}$ severe infectives and no removed (recovered) individuals. Let $(\Omega, \mathcal{F}, P)$ be a probability space on which are defined the following independent sets of random quantities:

(i) mild infectious careers $\mathcal{H}_{i}^{\mathrm{M}}=\left(I_{i}^{\mathrm{M}}, \eta_{i}^{\mathrm{MM}}, \eta_{i}^{\mathrm{MS}}, \eta_{i}^{\mathrm{MR}}, \xi_{i}^{\mathrm{MR}}\right)\left(i=-\left(m_{\mathrm{M}}-1\right),-\left(m_{\mathrm{M}}-2\right), \ldots\right)$, independent and identically distributed according to $\mathcal{H}^{\mathrm{M}}=\left(I^{\mathrm{M}}, \eta^{\mathrm{MM}}, \eta^{\mathrm{MS}}, \eta^{\mathrm{MR}}, \xi^{\mathrm{MR}}\right)$, where the components of $\mathcal{H}^{\mathrm{M}}$ are independent, $I^{\mathrm{M}}$ is exponentially distributed with mean $\gamma_{\mathrm{M}}^{-1}$, and $\eta^{\mathrm{MM}}, \eta^{\mathrm{MS}}, \eta^{\mathrm{MR}}$ and $\xi^{\mathrm{MR}}$ are homogeneous Poisson processes on $[0, \infty)$ having rates $\lambda_{\mathrm{M}} p_{\mathrm{MM}}, \lambda_{\mathrm{M}} p_{\mathrm{MS}}, \lambda_{\mathrm{M}} \alpha_{\mathrm{M}}$ and $\lambda_{\mathrm{M}} \alpha_{\mathrm{M}}$, respectively;

(ii) severe infectious careers $\mathcal{H}_{i}^{\mathrm{S}}=\left(I_{i}^{\mathrm{S}}, \eta_{i}^{\mathrm{SM}}, \eta_{i}^{\mathrm{SS}}, \eta_{i}^{\mathrm{SR}}, \xi_{i}^{\mathrm{SR}}\right)\left(i=-\left(m_{\mathrm{S}}-1\right),-\left(m_{\mathrm{S}}-2\right), \ldots\right)$, independent and identically distributed according to $\mathcal{H}^{\mathrm{S}}=\left(I^{\mathrm{S}}, \eta^{\mathrm{SM}}, \eta^{\mathrm{SS}}, \eta^{\mathrm{SR}}, \xi^{\mathrm{SR}}\right)$, where the components of $\mathcal{H}^{\mathrm{S}}$ are independent, $I^{\mathrm{S}}$ is exponentially distributed with mean $\gamma_{\mathrm{S}}^{-1}$, and $\eta^{\mathrm{SM}}, \eta^{\mathrm{SS}}, \eta^{\mathrm{SR}}$ and $\xi^{\mathrm{SR}}$ are are homogeneous Poisson processes on $[0, \infty)$ having rates $\lambda_{\mathrm{S}} p_{\mathrm{SM}}, \lambda_{\mathrm{S}} p_{\mathrm{SS}}, \lambda_{\mathrm{S}} \alpha_{\mathrm{S}}$ and $\lambda_{\mathrm{S}} \alpha_{\mathrm{S}}$, respectively;

(iii) $\chi_{i}^{(n)}(n=1,2, \ldots ; i=1,2, \ldots)$, where for each $n=1,2, \ldots, \chi_{1}^{(n)}, \chi_{2}^{(n)}, \ldots$ are independent and uniformly distributed on $\{1,2, \ldots, n\}$;

(iv) $\tilde{\chi}_{i}(i=1,2, \ldots)$, independent and uniformly distributed on $\left\{-\left(m_{\mathrm{M}}-1\right),-\left(m_{\mathrm{M}}-\right.\right.$ $2), \ldots, 0\}$.

The random quantities (iv) are required only if $m_{\mathrm{M}}>0$.

For $n=1,2, \ldots$, a realisation of the epidemic $E^{(n)}$ is constructed as follows. Label the $n$ suscpetibles $1,2, \ldots, n$, the $m_{\mathrm{M}}$ initial mild infectives $-\left(m_{\mathrm{M}}-1\right),-\left(m_{\mathrm{M}}-2\right), \ldots, 0$ and the 


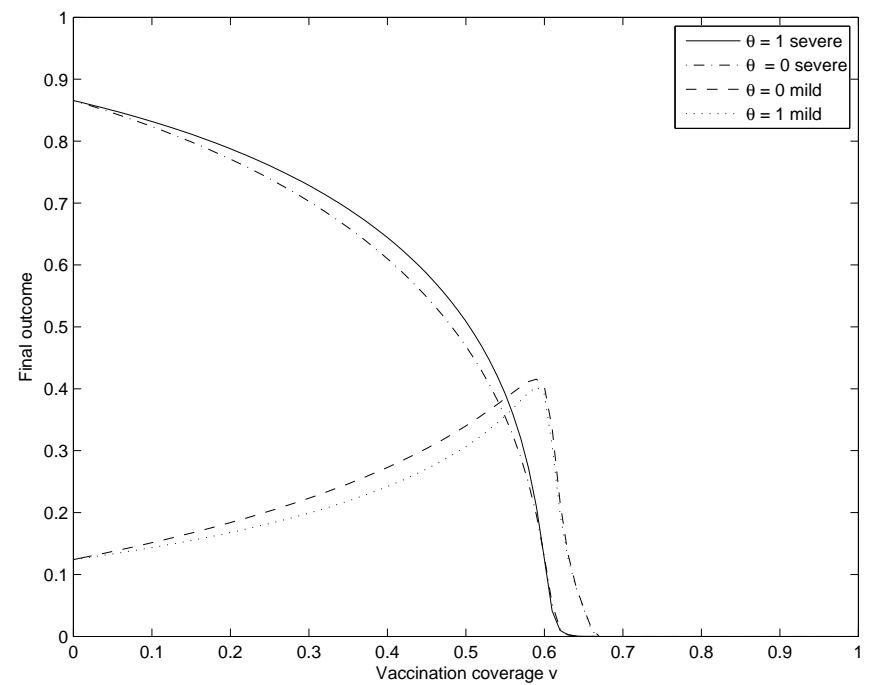

Figure 6: Final fractions infected as a function of the vaccination coverage for two choices of the non-random vaccine model.

$m_{\mathrm{S}}$ initial severe infectives $-\left(m_{\mathrm{M}}+m_{\mathrm{S}}-1\right),-\left(m_{\mathrm{M}}+m_{\mathrm{S}}-2\right), \ldots,-m_{\mathrm{M}}$. For $i=-\left(m_{\mathrm{S}}-\right.$ $1),-\left(m_{\mathrm{S}}-2\right), \ldots, 0$, the initial severe infective $i-m_{\mathrm{M}}$ has infectious period $I_{i}^{\mathrm{S}}$, during which it makes mild, severe and reinfection contacts with initial susceptibles at the points of $\eta_{i}^{\mathrm{SM}}, \eta_{i}^{\mathrm{SS}}$ and $\eta_{i}^{\mathrm{SR}}$, respectively, and reinfection contacts with initial mild infectives at the points of $n m_{\mathrm{M}}^{-1} \xi^{\mathrm{SR}}$. (If $\xi$ is a simple point process on $[0, \infty)$ with points at $t_{1}<t_{2}<\ldots$ and $\alpha>0$ then $\alpha \xi$ denotes the point process with points at $\alpha t_{1}<\alpha t_{2}<\ldots$; thus $n m_{\mathrm{M}}^{-1} \xi_{i}^{\mathrm{SR}}$ is a homogeneous Poisson process with rate $\left.n^{-1} m_{\mathrm{M}} \lambda_{\mathrm{S}} \alpha_{\mathrm{S}}\right)$. Similarly, for $i=-\left(m_{\mathrm{M}}-1\right),-\left(m_{\mathrm{M}}-2\right), \ldots, 0$, unless it is reinfected (see below), the initial mild infective $i$ has infectious period $I_{i}^{\mathrm{M}}$, during which it makes mild, severe and reinfection contacts with initial susceptibles at the points of $\eta_{i}^{\mathrm{MM}}, \eta_{i}^{\mathrm{MS}}$ and $\eta_{i}^{\mathrm{MR}}$, respectively, and reinfection contacts with initial mild infectives at the points of $n m_{\mathrm{M}}^{-1} \xi_{i}^{\mathrm{MR}}$.

For $k=1,2, \ldots$, the $k$ th contact made with the initial susceptibles is with individual $\chi_{k}^{(n)}$. If it is a mild or severe contact then the contacted individual becomes infected with the corresponding type if it is still susceptible, otherwise the the contact is ignored. If it is a reinfection contact then the contacted individual becomes a severe infective if it is a mild infective and the contacted individual is distinct from the individual making the contact, otherwise the contact is ignored. For $l=1,2, \ldots$, the $l$ th reinfection contact made with the initial mild infectives is with individual $\tilde{\chi}_{l}$. If the contacted individual is still mildly infected and distinct from the individual making the contact then it becomes severely infected, otherwise the contact is ignored. The $k$ th susceptible to be mildly infected in $E^{(n)}$ adopts the mild infectious career $\mathcal{H}_{k}^{\mathrm{M}}$. Suppose that this individual is infected at time $t_{1}$. Then, unless it is reinfected, it makes contacts during $\left[t_{1}, t_{1}+I_{k}^{\mathrm{M}}\right]$ at times given by $\left\{t_{1}+\eta_{k}^{\mathrm{MM}}\right\} \cup\left\{t_{1}+\right.$ $\left.\eta_{k}^{\mathrm{MS}}\right\} \cup\left\{t_{1}+\eta_{k}^{\mathrm{MR}}\right\} \cup\left\{t_{1}+n m_{\mathrm{M}}^{-1} \xi_{k}^{\mathrm{MR}}\right\}$. The $l$ th individual to become severely infected after 
time $t=0$ in $E^{(n)}$, either directly or by reinfection, adopts the severe infectious career $\mathcal{H}_{l}^{S}$. Suppose that this individual becomes severely infected at time $t_{2}$. Then it makes contacts during $\left[t_{2}, t_{2}+I_{l}^{\mathrm{S}}\right]$ at times given by $\left\{t_{2}+\eta_{l}^{\mathrm{SM}}\right\} \cup\left\{t_{2}+\eta_{l}^{\mathrm{SS}}\right\} \cup\left\{t_{2}+\eta_{l}^{\mathrm{SR}}\right\} \cup\left\{t_{2}+n m_{\mathrm{M}}^{-1} \xi_{l}^{\mathrm{SR}}\right\}$. When a mild infected is reinfected, its mild infectious period terminates immediately and it adopts a severe infectious career as described above. An individual becomes recovered and immune to further infection when it is no longer infectious. The epidemic stops when there is no infective, mild or severe, left in the population. It is easily verified that the epidemic $E^{(n)}$ is probabilistically equivalent to the IEDS model defined in Section 2.1.

The above random quantities can also be used to construct a realisation of a two-type (mild and severe) branching process, having initially $m_{\mathrm{M}}$ mild indivdiuals, labelled $-\left(m_{\mathrm{M}}-1\right),-\left(m_{\mathrm{M}}-\right.$ $2), \ldots, 0$, and $m_{\mathrm{S}}$ severe individuals, labelled $-\left(m_{\mathrm{M}}+m_{\mathrm{S}}-1\right),-\left(m_{\mathrm{M}}+m_{\mathrm{S}}-2\right), \ldots,-m_{\mathrm{M}}$. For $i=-\left(m_{\mathrm{M}}-1\right),-\left(m_{\mathrm{M}}-2\right), \ldots, 0$, the initial mild individual $i$ lives until time $I_{i}^{\mathrm{M}}$, has one mild child at each of the times given by $\eta_{i}^{\mathrm{MM}} \cap\left[0, I_{i}^{\mathrm{M}}\right]$ and one severe child at each of the times given by $\eta_{i}^{\mathrm{MS}} \cap\left[0, I_{i}^{\mathrm{M}}\right]$. Similarly, for $i=-\left(m_{\mathrm{S}}-1\right),-\left(m_{\mathrm{S}}-2\right), \ldots, 0$, the initial severe individual $i-m_{\mathrm{M}}$ lives until time $I_{i}^{\mathrm{S}}$, has one mild child at each of the times given by $\eta_{i}^{\mathrm{SM}} \cap\left[0, I_{i}^{\mathrm{S}}\right]$ and one severe child at each of the times given by $\eta_{i}^{\mathrm{SS}} \cap\left[0, I_{i}^{\mathrm{S}}\right]$. For $k=1,2, \ldots$, the $k$ th mild individual born in $(0, \infty)$ in the branching process lives until age $I_{k}^{\mathrm{M}}$, has one mild child at each of the times given by $\left\{t_{k}^{\mathrm{M}}+\eta_{k}^{\mathrm{MM}}\right\} \cap\left[0, I_{k}^{\mathrm{M}}\right]$ and one severe child at each of the times given by $\left\{t_{k}^{\mathrm{M}}+\eta_{k}^{\mathrm{MS}}\right\} \cap\left[0, I_{k}^{\mathrm{M}}\right]$, where $t_{k}^{\mathrm{M}}$ is the time when the $k$ th mild birth occurs. Similarly, for $l=1,2, \ldots$, if the $l$ th severe individual is born at time $t_{l}^{\mathrm{S}}>0$, then she has one mild child at each of the times given by $\left\{t_{l}^{\mathrm{S}}+\eta_{l}^{\mathrm{SM}}\right\} \cap\left[0, I_{k}^{\mathrm{S}}\right]$ and one severe child at each of the times given by $\left\{t_{l}^{\mathrm{S}}+\left\{\eta_{l}^{\mathrm{SS}}\right\} \cap\left[0, I_{k}^{\mathrm{S}}\right]\right.$. Of course, the branching process stops if there is no live individual.

For $n=1,2, \ldots$, let

$$
M_{n}=\min \left\{k \geq 2: \chi_{1}^{(n)}, \chi_{2}^{(n)}, \ldots, \chi_{k}^{(n)} \text { are not distinct }\right\}
$$

Then, noting the connection with the 'birthday problem', as in the proof of Ball and Donnelly [22], Theorem 2.1, we may assume that

$$
n^{-1 / 2} M_{n} \stackrel{\text { a.s. }}{\longrightarrow} M \quad \text { as } \quad n \rightarrow \infty,
$$

where $\stackrel{\text { a.s. }}{\longrightarrow}$ denotes almost sure convergence and $M$ is a strictly positive random variable defined on $(\Omega, \mathcal{F}, P)$ having probability density function $f(x)=x \exp \left(-\frac{1}{2} x^{2}\right)(x>0)$.

For $t \geq 0$, let $\boldsymbol{Y}(t)=\left(Y_{\mathrm{M}}(t), Y_{\mathrm{S}}(t)\right)$, where $Y_{\mathrm{M}}(t)$ and $Y_{\mathrm{S}}(t)$ denote respectively the numbers of mild and severe individuals alive in the branching process at time $t$, and let $\boldsymbol{Y}^{(n)}(t)=$ $\left(Y_{\mathrm{M}}^{(n)}(t), Y_{\mathrm{S}}^{(n)}(t)\right)$, where $Y_{\mathrm{M}}^{(n)}(t)$ and $Y_{\mathrm{S}}^{(n)}(t)$ denote respectively the numbers of mild and severe infectives at time $t$ in the epidemic $E^{(n)}(n=1,2, \ldots)$. Further, for $t \geq 0$, let $Z_{\mathrm{M}}(t)$ and $Z_{\mathrm{S}}(t)$ denote respectively the total numbers of mild and severe births during $(0, t]$ in the branching process. Suppose that $p_{\mathrm{MS}} p_{\mathrm{SM}}>0$. Then the branching process is indecomposable (see e.g. Haccou et al. [16], page 27) and its Malthusian parameter, $\alpha$ say, is given by the 
maximal eigenvalue of the matrix

$$
G=\left[\begin{array}{ll}
\lambda_{\mathrm{M}} p_{\mathrm{MM}}-\gamma_{\mathrm{M}} & \lambda_{\mathrm{M}} p_{\mathrm{MS}} \\
\lambda_{\mathrm{S}} p_{\mathrm{SM}} & \lambda_{\mathrm{S}} p_{\mathrm{SS}}-\gamma_{\mathrm{S}}
\end{array}\right]
$$

Further, let $\boldsymbol{v}=\left(v_{\mathrm{M}}, v_{\mathrm{S}}\right)$ be the left eigenvector of $G$ corresponding to $\alpha$, normalized so that $v_{\mathrm{M}}+v_{\mathrm{S}}=1$. Then Theorem 2 on page 206 of Athreya and Ney [23], together with the theory of asymptotic growth and stabilisation of general multitype branching processes (e.g. Jagers [24]), implies that there exists a nonnegative random variable $W$, defined on $(\Omega, \mathcal{F}, P)$, so that almost surely

$$
\lim _{t \rightarrow \infty} \mathrm{e}^{-\alpha t} Z_{\mathrm{M}}(t)=\alpha^{-1} \lambda_{\mathrm{M}} v_{\mathrm{M}} W \quad \text { and } \quad \lim _{t \rightarrow \infty} \mathrm{e}^{-\alpha t} Z_{\mathrm{S}}(t)=\alpha^{-1} \lambda_{\mathrm{S}} v_{\mathrm{S}} W
$$

Moreover, $W(\omega)=0$ if and only if $\omega \in A_{\mathrm{EXT}}$, where $A_{\mathrm{EXT}} \in \mathcal{F}$ is the set on which the branching process becomes extinct.

Theorem 5.1 (a) For P-almost all $\omega \in A_{E X T}$,

$$
\lim _{n \rightarrow \infty} \sup _{0 \leq t<\infty}\left|\boldsymbol{Y}^{(n)}(t, \omega)-\boldsymbol{Y}(t, \omega)\right|=0
$$

(b) For P-almost all $\omega \in \Omega \backslash A_{E X T}$,

$$
\lim _{n \rightarrow \infty} \sup _{0 \leq t \leq \beta \log n}\left|\boldsymbol{Y}^{(n)}(t, \omega)-\boldsymbol{Y}(t, \omega)\right|=0,
$$

for any $\beta \in\left[0,(2 \alpha)^{-1}\right)$.

Proof: $\quad$ Let $C_{i}^{\mathrm{M}}=\left|\left(\eta_{i}^{\mathrm{MM}} \cup \eta_{i}^{\mathrm{MS}} \cup \eta_{i}^{\mathrm{MR}}\right) \cap\left[0, I_{i}^{\mathrm{M}}\right]\right|\left(i=-\left(m_{\mathrm{M}}-1\right),-\left(m_{\mathrm{M}}-2\right), \ldots\right)$ and $C_{i}^{\mathrm{S}}=\left|\left(\eta_{i}^{\mathrm{SM}} \cup \eta_{i}^{\mathrm{SS}} \cup \eta_{i}^{\mathrm{SR}}\right) \cap\left[0, I_{i}^{\mathrm{S}}\right]\right|\left(i=-\left(m_{\mathrm{S}}-1\right),-\left(m_{\mathrm{S}}-2\right), \ldots\right)$, so, for example, $C_{0}^{\mathrm{M}}$ is the total number of contacts with the initial suscepibles made by the initial mild infective labelled 0 . In view of (5.1), there exists $B_{1} \in \mathcal{F}$ with $P\left(B_{1}\right)=1$ such that $\lim _{n \rightarrow \infty} n^{-1 / 2} M_{n}(\omega)=M(\omega)$ for all $\omega \in B_{1}$. Fix $\omega \in A_{\mathrm{EXT}} \cap B_{1}$, so $Z_{\mathrm{M}}(\infty, \omega)$ and $Z_{\mathrm{S}}(\infty, \omega)$ are both finite. Hence $\sum_{i=-\left(m_{\mathrm{M}}-1\right)}^{Z_{\mathrm{M}}(\infty, \omega)} C_{i}^{\mathrm{M}}(\omega)$ and $\sum_{i=-(m-1)}^{Z(\infty, \omega)} C_{i}^{\mathrm{S}}(\omega)$ are also both finite. Now $M_{n}(\omega) \rightarrow \infty$ as $n \rightarrow \infty$, so there exists an integer $N_{1}(\omega)$ such that, for all $n \geq N_{1}(\omega)$, all the contacts with initial susceptibles in $E^{(n)}$ are with distinct individuals. For $i=-\left(m_{\mathrm{M}}-1\right),-\left(m_{\mathrm{M}}-2\right), \ldots$, let $\tau_{i}^{\mathrm{MR}}(\omega)$ be the time of the first point of $\xi_{i}^{\mathrm{MR}}(\omega)$ in $(0, \infty)$ and, for $i=-\left(m_{\mathrm{S}}-1\right),-\left(m_{\mathrm{S}}-2\right), \ldots$, define $\tau_{i}^{\mathrm{MS}}(\omega)$ similarly. Note that, for example, the $i$ th mild infective does not make any reinfection contact with the initial mild infectives if $n>m_{\mathrm{M}} I_{i}^{\mathrm{M}}(\omega) / \tau_{i}^{\mathrm{MR}}(\omega)$. Thus, if $N_{2}(\omega)$ is the smallest integer greater than both $\max \left\{m_{\mathrm{M}} I_{i}^{\mathrm{M}}(\omega) / \tau_{i}^{\mathrm{MR}}(\omega): i=-\left(m_{\mathrm{M}}-1\right),-\left(m_{\mathrm{M}}-2\right), \ldots, Z_{\mathrm{M}}(\infty, \omega)\right\}$ and $\max \left\{m_{\mathrm{S}} I_{i}^{\mathrm{S}}(\omega) / \tau_{i}^{\mathrm{SR}}(\omega): i=-\left(m_{\mathrm{S}}-1\right),-\left(m_{\mathrm{S}}-2\right), \ldots, Z_{\mathrm{S}}(\infty, \omega)\right\}$, then for all $n \geq N_{2}(\omega)$, there is no reinfection contact with the initial mild infectives in $E^{(n)}$. Hence, for all $n>$ $\max \left(N_{1}(\omega), N_{2}(\omega)\right)$, (i) every birth in the branching process yields a new infective in $E^{(n)}$ and (ii) there is no reinfection in $E^{(n)}$, and (5.4) follows since $P\left(A_{\mathrm{EXT}} \cap B_{1}\right)=P\left(A_{\mathrm{EXT}}\right)$. 
To prove part (b), first note that by the strong law of large numbers there exists $B_{2} \in \mathcal{F}$, with $P\left(B_{2}\right)=1$, such that $\lim _{n \rightarrow \infty} n^{-1} C_{i}^{\mathrm{M}}(\omega)=\lambda_{\mathrm{M}}\left(1+\alpha_{\mathrm{M}}\right) \gamma_{\mathrm{M}}^{-1}$ and $\lim _{n \rightarrow \infty} n^{-1} C_{i}^{\mathrm{S}}(\omega)=$ $\lambda_{\mathrm{S}}\left(1+\alpha_{\mathrm{S}}\right) \gamma_{\mathrm{S}}^{-1}$ for all $\omega \in B_{2}$. Also, (5.3) implies that there exists $B_{3} \in \mathcal{F}$, with $P\left(B_{3}\right)=1$, such that for all $\omega \in B_{3}, \lim _{t \rightarrow \infty} \mathrm{e}^{-\alpha t} Z_{\mathrm{M}}(t, \omega)=\alpha^{-1} \lambda_{\mathrm{M}} v_{\mathrm{M}} W(\omega)$ and $\lim _{t \rightarrow \infty} \mathrm{e}^{-\alpha t} Z_{\mathrm{S}}(t, \omega)=$ $\alpha^{-1} \lambda_{\mathrm{S}} v_{\mathrm{S}} W(\omega)$, with $W(\omega)>0$ if $\omega \in B_{3} \cap \backslash A_{\mathrm{EXT}}$. Fix $\omega \in\left(B_{1} \cap B_{2} \cap B_{3}\right) \backslash A_{\mathrm{EXT}}$ and $\beta \in\left(0,(2 \alpha)^{-1}\right)$. Since $\omega \in B_{3}$, there exists an integer $N_{3}(\omega)$ such that $Z_{\mathrm{M}}(\beta \log n, \omega)<$ $2 \alpha^{-1} \lambda_{\mathrm{M}} v_{\mathrm{M}} W(\omega) n^{\alpha \beta}$ and $Z_{\mathrm{S}}(\beta \log n, \omega)<2 \alpha^{-1} \lambda_{\mathrm{S}} v_{\mathrm{S}} W(\omega) n^{\alpha \beta}$ for all $n \geq N_{3}(\omega)$. Further, since $\omega \in B_{2}$, there exists an integer $N_{4}(\omega) \geq N_{3}(\omega)$ such that, for all $n \geq N_{4}(\omega)$,

$$
\sum_{i=-\left(m_{\mathrm{M}}-1\right)}^{Z_{\mathrm{M}}(\beta \log n, \omega)} C_{i}^{\mathrm{M}}(\omega)<\sum_{i=-\left(m_{\mathrm{M}}-1\right)}^{2 \alpha^{-1} \lambda_{\mathrm{M}} v_{\mathrm{M}} W(\omega) n^{\alpha \beta}} C_{i}^{\mathrm{M}}(\omega)<4 \alpha^{-1} \lambda_{\mathrm{M}}^{2} v_{\mathrm{M}}\left(1+\alpha_{\mathrm{M}}\right) \gamma_{\mathrm{M}}^{-1} W(\omega) n^{\alpha \beta}
$$

and

$$
\sum_{i=-\left(m_{\mathrm{S}}-1\right)}^{Z_{\mathrm{S}}(\beta \log n, \omega)} C_{i}^{\mathrm{S}}(\omega)<4 \alpha^{-1} \lambda_{\mathrm{S}}^{2} v_{\mathrm{S}}\left(1+\alpha_{\mathrm{S}}\right) \gamma_{\mathrm{S}}^{-1} W(\omega) n^{\alpha \beta}
$$

Also, as $\omega \in B_{1}$, there exists an integer $N_{5}(\omega)$ such that, for all $n \geq N_{5}(\omega)$,

$$
M_{n}(\omega)>\frac{1}{2} n^{\frac{1}{2}} M(\omega) .
$$

It follows from (5.6), (5.7) and (5.8) that there exists an integer $N_{6}(\omega)$ such that, for all $n \geq N_{6}(\omega)$,

$$
\sum_{i=-\left(m_{\mathrm{M}}-1\right)}^{Z_{\mathrm{M}}(\beta \log n, \omega)} C_{i}^{\mathrm{M}}(\omega)+\sum_{i=-\left(m_{\mathrm{S}}-1\right)}^{Z_{\mathrm{S}}(\beta \log n, \omega)} C_{i}^{\mathrm{S}}(\omega)<M_{n}(\omega),
$$

so, for such $n$, all contacts with the initial susceptibles during $[0, \beta \log n]$ in $E^{(n)}$ are with distinct individuals.

Let $I_{*}(\omega)=\max \left(I_{*}^{\mathrm{M}}(\omega), I_{*}^{\mathrm{S}}(\omega)\right)$, where

$$
I_{*}^{\mathrm{M}}(\omega)=\max _{-\left(m_{\mathrm{M}}-1\right) \leq i \leq 0} I_{i}^{\mathrm{M}}(\omega) \quad \text { and } \quad I_{*}^{\mathrm{S}}(\omega)=\max _{-\left(m_{\mathrm{S}}-1\right) \leq i \leq 0} I_{i}^{\mathrm{S}}(\omega),
$$

be the time of the last death of an initial individual in the branching process. Then, noting that $Z_{\mathrm{M}}\left(I_{*}(\omega), \omega\right)$ and $Z_{\mathrm{S}}\left(I_{*}(\omega), \omega\right)$ are both finite, arguing as in the proof of part (a) shows that there exists an integer $N_{7}(\omega)$ such that, for all $n \geq N_{7}(\omega)$, there is no reinfection contact with the initial mild infectives in $E^{(n)}$. Thus, recalling $(5.9), \boldsymbol{Y}^{(n)}(t, \omega)(0 \leq t \leq \beta \log n)$ and $\boldsymbol{Y}(t, \omega)(0 \leq t \leq \beta \log n)$ coincide for all $n \geq \max \left(N_{6}(\omega), N_{6}(\omega)\right)$ and (5.5) follows since $P\left(\left(B_{1} \cap B_{2} \cap B_{3}\right) \backslash A_{\mathrm{EXT}}\right)=P\left(\Omega \backslash A_{\mathrm{EXT}}\right)$.

When $p_{\mathrm{MS}} p_{\mathrm{SM}}=0$ the branching process is decomposable. It is readily seen that part (a) of Theorem 5.1 still holds in this case and that part (b) holds with $\alpha$ replaced by $\max \left(\lambda_{\mathrm{M}} p_{\mathrm{MM}}-\right.$ $\left.\gamma_{\mathrm{M}}, \lambda_{\mathrm{S}} p_{\mathrm{SS}}-\gamma_{\mathrm{S}}\right)$. The following corollary to Theorem 5.1 is immediate and holds without 
requiring $p_{\mathrm{MS}} p_{\mathrm{SM}}>0$. Recall that $Z_{\mathrm{M}}(\infty)$ and $Z_{\mathrm{S}}(\infty)$ are respectively the total numbers of mild and severe births that ever occur in the branching process. For $n=1,2, \ldots$, let $Z_{\mathrm{M}}^{(n)}(\infty)$ and $Z_{\mathrm{S}}^{(n)}(\infty)$ be respectively the total number of mild and severe removals in the epidemic $E^{(n)}$. Note that $Z_{\mathrm{M}}(\infty)$ and $Z_{\mathrm{S}}(\infty)$ may be infinite but $Z_{\mathrm{M}}^{(n)}(\infty)$ and $Z_{\mathrm{S}}^{(n)}(\infty)$ are necessarily finite.

Corollary 5.2 (a) For P-almost all $\omega \in A_{E X T}$,

$$
\lim _{n \rightarrow \infty}\left(Z_{\mathrm{M}}^{(n)}(\infty, \omega), Z_{\mathrm{S}}^{(n)}(\infty, \omega)\right)=\left(Z_{\mathrm{M}}(\infty, \omega)+m_{\mathrm{M}}, Z_{\mathrm{S}}^{(n)}(\infty, \omega)+m_{\mathrm{S}}\right) .
$$

(b) For P-almost all $\omega \in \Omega \backslash A_{E X T}$,

$$
\lim _{n \rightarrow \infty} Z_{\mathrm{M}}^{(n)}(\infty, \omega)+Z_{\mathrm{S}}^{(n)}(\infty, \omega)=\infty
$$

\subsection{LLN and CLT for final size distribution}

As in Section 5.1, consider a sequence of IEDS epidemics, $\left\{E^{(n)}: n \geq 1\right\}$ say, indexed by the initial number of susceptibles $n$. For $t \geq 0$, let $X^{(n)}(t), Y_{\mathrm{M}}^{(n)}(t), Y_{\mathrm{S}}^{(n)}(t), Z_{\mathrm{M}}^{(n)}(t)$ and $Z_{\mathrm{S}}^{(n)}(t)$ denote respectively the numbers of susceptible, mild infective, severe infective, mild removed and severe removed individuals at time $t$. Suppose that $Y_{\mathrm{M}}^{(n)}(0)=m_{\mathrm{M}}^{(n)}, Y_{\mathrm{S}}^{(n)}(0)=$ $m_{\mathrm{S}}^{(n)}, Z_{\mathrm{M}}^{(n)}(0)=0$ and $Z_{\mathrm{S}}^{(n)}(0)=0(n=1,2, \ldots)$, so initially there are $m_{\mathrm{M}}^{(n)}$ mild infectives and $m_{\mathrm{S}}^{(n)}$ severe infectives in $E^{(n)}$. The epidemics $E^{(n)}(n=1,2, \ldots)$ each have the same infection, reinfection and removal parameters. The process $\boldsymbol{X}^{(n)}=\left\{\boldsymbol{X}^{(n)}(t): t \geq 0\right\}$, where $\boldsymbol{X}^{(n)}(t)=\left(X^{(n)}(t), Y_{\mathrm{M}}^{(n)}(t), Y_{\mathrm{S}}^{(n)}(t), Z_{\mathrm{M}}^{(n)}(t), Z_{\mathrm{S}}^{(n)}(t)\right)^{\top}(t \geq 0)$ is a continuous-time Markov chain with transition rates

$$
q_{\left(s, i_{\mathrm{M}}, i_{\mathrm{S}}, r_{\mathrm{M}}, r_{\mathrm{S}}\right),\left(s-1, i_{\mathrm{M}}+1, i_{\mathrm{S}}, r_{\mathrm{M}}, r_{\mathrm{S}}\right)}^{(n)} n^{-1}\left[\lambda_{\mathrm{M}} p_{\mathrm{MM}} i_{\mathrm{M}}+\lambda_{\mathrm{S}} p_{\mathrm{SM}} i_{\mathrm{S}}\right]
$$

for a new mild infection,

$$
q_{\left(s, i_{\mathrm{M}}, i_{\mathrm{S}}, r_{\mathrm{M}}, r_{\mathrm{S}}\right),\left(s-1, i_{\mathrm{M}}, i_{\mathrm{S}}+1, r_{\mathrm{M}}, r_{\mathrm{S}}\right)}^{(-1} n^{-1}\left[\lambda_{\mathrm{M}} p_{\mathrm{MS}} i_{\mathrm{M}}+\lambda_{\mathrm{S}} p_{\mathrm{SS}} i_{\mathrm{S}}\right]
$$

for a new severe infection,

$$
q_{\left(s, i_{\mathrm{M}}, i_{\mathrm{S}}, r_{\mathrm{M}}, r_{\mathrm{S}}\right),\left(s, i_{\mathrm{M}}-1, i_{\mathrm{S}}+1, r_{\mathrm{M}}, r_{\mathrm{S}}\right)}^{(n)} n^{-1} i_{\mathrm{M}}\left[\lambda_{\mathrm{M}} \alpha_{\mathrm{M}}\left(i_{\mathrm{M}}-1\right)+\lambda_{\mathrm{S}} \alpha_{\mathrm{S}} i_{\mathrm{S}}\right]
$$

for a reinfection,

$$
q_{\left(s, i_{\mathrm{M}}, i_{\mathrm{S}}, r_{\mathrm{M}}, r_{\mathrm{S}}\right),\left(s, i_{\mathrm{M}}-1, i_{\mathrm{S}}, r_{\mathrm{M}}+1, r_{\mathrm{S}}\right)}^{(n)} \gamma_{\mathrm{M}} i_{\mathrm{M}},
$$

for a mild removal, and

$$
q_{\left(s, i_{\mathrm{M}}, i_{\mathrm{S}}, r_{\mathrm{M}}, r_{\mathrm{S}}\right),\left(s, i_{\mathrm{M}}, i_{\mathrm{S}}-1, r_{\mathrm{M}}, r_{\mathrm{S}}+1\right)}^{(n)} \gamma_{\mathrm{S}} i_{\mathrm{S}}
$$


for a severe removal, where $\left(s, i_{\mathrm{M}}, i_{\mathrm{S}}, r_{\mathrm{M}}, r_{\mathrm{S}}\right)^{\top}$ is the state of $\boldsymbol{X}^{(n)}$ at a given time. The factor $\left(i_{\mathrm{M}}-1\right)$ in (5.10) arises because a mild infective cannot reinfect itself.

Let $\boldsymbol{i}=\left(s, i_{\mathrm{M}}, i_{\mathrm{S}}, r_{\mathrm{M}}, r_{\mathrm{S}}\right)^{\top}$ and note that each of the above transition rates admits the form $n\left[f\left(n^{-1} \boldsymbol{i}\right)+O\left(n^{-1}\right)\right]$ as $n \rightarrow \infty$, where $f$ depends on the type of transition. Thus $\left\{\boldsymbol{X}^{(n)}: n \geq\right.$ $1\}$ satisfies the more general form of a density dependent population process defined by Ethier and Kurtz [17], Chapter 11, Equation (1.13). (Apart from those for reinfection, the transition rates satisfy the usual density dependent form in which the $O\left(n^{-1}\right)$ term is identically zero.)

Suppose further that $n^{-1} m_{\mathrm{M}}^{(n)} \rightarrow \mu_{\mathrm{M}}$ and $n^{-1} m_{\mathrm{S}}^{(n)} \rightarrow \mu_{\mathrm{S}}$ as $n \rightarrow \infty$, where $\mu_{\mathrm{M}}+\mu_{\mathrm{S}}>$ 0 . Then, Ethier and Kurtz [17], Theorem 11.2.1, implies that as $n \rightarrow \infty, n^{-} \boldsymbol{X}^{(n)}$ converges almost surely over any finite time interval to $\boldsymbol{x}=\{\boldsymbol{x}(t): t \geq 0\}$, where $\boldsymbol{x}(t)=$ $\left(x(t), y_{\mathrm{M}}(t), y_{\mathrm{S}}(t), z_{\mathrm{M}}(t), z_{\mathrm{S}}(t)\right)^{\top}$ and $\left(x(t), y_{\mathrm{M}}(t), y_{\mathrm{S}}(t), z_{\mathrm{M}}(t), z_{\mathrm{S}}(t)\right)$ is given by the solution of (3.6) with initial condition (3.7). Moreover, a central limit theorem for fluctuations of $\boldsymbol{X}^{(n)}$ about $\boldsymbol{x}$ is given by Ethier and Kurtz [17], Theorem 11.2.3. (In this subsection is more convenient for $\boldsymbol{x}(t)$ to be a column vector, rather than a row vector as it is in Section 3.2.)

We are primarily interested in the final outcome of the epidemic $E^{(n)}$, which is not covered by the above asymptotic results. For $n \geq 1$, the final outcome of $E^{(n)}$ is given by $\boldsymbol{X}^{(n)}\left(\tau^{(n)}\right)$, where $\tau^{(n)}=\inf \left\{t>0: Y_{\mathrm{M}}^{(n)}(t)+Y_{\mathrm{S}}^{(n)}(t)=0\right\}$ is the duration of the epidemic $E^{(n)}$. A central limit theorem for $\boldsymbol{X}^{(n)}\left(\tau^{(n)}\right)$ cannot be obtained directly from the theory in Ethier and Kurtz [17] because $\inf \left\{t>0: y_{\mathrm{M}}(t)+y_{\mathrm{S}}(t) \leq 0\right\}=\infty$. Thus, as in Ball and Britton [4], we consider the following random time scale transformation of $\boldsymbol{X}^{(n)}$, cf. Ethier and Kurtz [17], page 467.

For $t \in\left[0, \tau^{(n)}\right]$, let $A^{(n)}(t)=\int_{0}^{t} n^{-1}\left(\lambda_{\mathrm{M}} Y_{\mathrm{M}}^{(n)}(u)+\lambda_{\mathrm{S}} Y_{\mathrm{S}}^{(n)}(u)\right) d u$ be the total force of infection exerted on a given susceptible in $E^{(n)}$ during $[0, t]$, and let $A^{(n)}=A^{(n)}\left(\tau^{(n)}\right)$. For $0 \leq$ $t \leq A^{(n)}$, let $U^{(n)}(t)=\inf \left\{u \geq 0: A^{(n)}(u)=t\right\}$, let $\tilde{\boldsymbol{X}}^{(n)}(t)=\boldsymbol{X}^{(n)}\left(U^{(n)}(t)\right)$ and write $\tilde{\boldsymbol{X}}^{(n)}(t)=\left(\tilde{X}^{(n)}(t), \tilde{Y}_{\mathrm{M}}^{(n)}(t), \tilde{Y}_{\mathrm{S}}^{(n)}(t), \tilde{Z}_{\mathrm{M}}^{(n)}(t), \tilde{Z}_{\mathrm{S}}^{(n)}(t)\right)^{\top}$. The process $\tilde{\boldsymbol{X}}^{(n)}=\left\{\tilde{\boldsymbol{X}}^{(n)}(t): 0 \leq\right.$ $\left.t \leq A^{(n)}\right\}$ is obtained from $\boldsymbol{X}^{(n)}$ by running the clock at rate $n\left(\lambda_{\mathrm{M}} Y_{\mathrm{M}}^{(n)}(u)+\lambda_{\mathrm{S}} Y_{\mathrm{S}}^{(n)}(u)\right)^{-1}$. Thus the transition rates of $\tilde{\boldsymbol{X}}^{(n)}$ are obtained from those given above for $\boldsymbol{X}^{(n)}$ by dividing by $\lambda_{\mathrm{M}} i_{\mathrm{M}}+\lambda_{\mathrm{S}} i_{\mathrm{S}}$.

The possible jumps of $\tilde{\boldsymbol{X}}^{(n)}$ from a typical state $\boldsymbol{i}=\left(s, i_{\mathrm{M}}, i_{\mathrm{S}}, r_{\mathrm{M}}, r_{\mathrm{S}}\right)^{\top}$ are $\Delta=\left\{(-1,1,0,0,0)^{\top}\right.$, $\left.(-1,0,1,0,0)^{\top},(0,-1,1,0,0),(0,-1,0,1,0)^{\top},(0,0,-1,0,1)^{\top}\right\}$. The rates of these jumps admit the form $n\left[\tilde{\beta}_{\boldsymbol{l}}\left(n^{-1} \boldsymbol{i}\right)+O\left(n^{-1}\right)\right](\boldsymbol{l} \in \Delta)$, as $n \rightarrow \infty$, where the functions $\tilde{\beta}_{\boldsymbol{l}}(\boldsymbol{l} \in \Delta)$ are 
given by

$$
\begin{aligned}
& \tilde{\beta}_{(-1,1,0,0,0)}\left(\tilde{x}, \tilde{y}_{\mathrm{M}}, \tilde{y}_{\mathrm{S}}, \tilde{z}_{\mathrm{M}}, \tilde{z}_{\mathrm{S}}\right)=\frac{\tilde{x}\left(\lambda_{\mathrm{M}} p_{\mathrm{MM}} \tilde{y}_{\mathrm{M}}+\lambda_{\mathrm{S}} p_{\mathrm{SM}} \tilde{y}_{\mathrm{S}}\right)}{\lambda_{\mathrm{M}} \tilde{y}_{\mathrm{M}}+\lambda_{\mathrm{S}} \tilde{y}_{\mathrm{S}}}, \\
& \tilde{\beta}_{(-1,0,1,0,0)}\left(\tilde{x}, \tilde{y}_{\mathrm{M}}, \tilde{y}_{\mathrm{S}}, \tilde{z}_{\mathrm{M}}, \tilde{z}_{\mathrm{S}}\right)=\frac{\tilde{x}\left(\lambda_{\mathrm{M}} p_{\mathrm{MS}} \tilde{y}_{\mathrm{M}}+\lambda_{\mathrm{S}} p_{\mathrm{SS}} \tilde{y}_{\mathrm{S}}\right)}{\lambda_{\mathrm{M}} \tilde{y}_{\mathrm{M}}+\lambda_{\mathrm{S}} \tilde{y}_{\mathrm{S}}}, \\
& \tilde{\beta}_{(0,-1,1,0,0)}\left(\tilde{x}, \tilde{y}_{\mathrm{M}}, \tilde{y}_{\mathrm{S}}, \tilde{z}_{\mathrm{M}}, \tilde{z}_{\mathrm{S}}\right)=\frac{\tilde{y}_{\mathrm{M}}\left(\lambda_{\mathrm{M}} \alpha_{\mathrm{M}} \tilde{y}_{\mathrm{M}}+\lambda_{\mathrm{S}} \alpha_{\mathrm{S}} \tilde{y}_{\mathrm{S}}\right)}{\lambda_{\mathrm{M}} \tilde{y}_{\mathrm{M}}+\lambda_{\mathrm{S}} \tilde{y}_{\mathrm{S}}} \\
& \tilde{\beta}_{(0,-1,0,1,0)}\left(\tilde{x}, \tilde{y}_{\mathrm{M}}, \tilde{y}_{\mathrm{S}}, \tilde{z}_{\mathrm{M}}, \tilde{z}_{\mathrm{S}}\right)=\frac{\gamma_{\mathrm{M}} \tilde{y}_{\mathrm{M}}}{\lambda_{\mathrm{M}} \tilde{y}_{\mathrm{M}}+\lambda_{\mathrm{S}} \tilde{y}_{\mathrm{S}}} \\
& \tilde{\beta}_{(0,0,-1,0,1)}\left(\tilde{x}, \tilde{y}_{\mathrm{M}}, \tilde{y}_{\mathrm{S}}, \tilde{z}_{\mathrm{M}}, \tilde{z}_{\mathrm{S}}\right)=\frac{\gamma_{\mathrm{S}} \tilde{y}_{\mathrm{S}}}{\lambda_{\mathrm{M}} \tilde{y}_{\mathrm{M}}+\lambda_{\mathrm{S}} \tilde{y}_{\mathrm{S}}} .
\end{aligned}
$$

Let

$$
\tilde{F}\left(\tilde{x}, \tilde{y}_{\mathrm{M}}, \tilde{y}_{\mathrm{S}}, \tilde{z}_{\mathrm{M}}, \tilde{z}_{\mathrm{S}}\right)=\sum_{\boldsymbol{l} \in \Delta} \tilde{\beta}_{\boldsymbol{l}}\left(\tilde{x}, \tilde{y}_{\mathrm{M}}, \tilde{y}_{\mathrm{S}}, \tilde{z}_{\mathrm{M}}, \tilde{z}_{\mathrm{S}}\right) \boldsymbol{l}
$$

and, for $t \geq 0$, let $\tilde{\boldsymbol{x}}(t)=\left(\tilde{x}(t), \tilde{y}_{\mathrm{M}}(t), \tilde{y}_{\mathrm{S}}(t), \tilde{z}_{\mathrm{M}}(t), \tilde{z}_{\mathrm{S}}(t)\right)^{\top}$ be defined by

$$
\tilde{\boldsymbol{x}}(t)=\tilde{\boldsymbol{x}}(0)+\int_{0}^{t} \tilde{F}(\tilde{\boldsymbol{x}}(u)) d u
$$

where $\tilde{\boldsymbol{x}}(0)=\left(1, \mu_{\mathrm{M}}, \mu_{\mathrm{S}}, 0,0\right)^{\top}$. Thus $\tilde{\boldsymbol{x}}(t)$ satisfies the differential equation

$$
\begin{aligned}
\frac{d \tilde{x}}{d t} & =-\tilde{x} \\
\frac{d \tilde{y}_{\mathrm{M}}}{d t} & =\frac{\tilde{x}\left(\lambda_{\mathrm{M}} p_{\mathrm{MM}} \tilde{y}_{\mathrm{M}}+\lambda_{\mathrm{S}} p_{\mathrm{SM}} \tilde{y}_{\mathrm{S}}\right)-\left(\lambda_{\mathrm{M}} \alpha_{\mathrm{M}} \tilde{y}_{\mathrm{M}}+\lambda_{\mathrm{S}} \alpha_{\mathrm{S}} \tilde{y}_{\mathrm{S}}+\gamma_{\mathrm{M}}\right) \tilde{y}_{\mathrm{M}}}{\lambda_{\mathrm{M}} \tilde{y}_{\mathrm{M}}+\lambda_{\mathrm{S}} \tilde{y}_{\mathrm{S}}} \\
\frac{d \tilde{y}_{\mathrm{S}}}{d t} & =\frac{\tilde{x}\left(\lambda_{\mathrm{M}} p_{\mathrm{MS}} \tilde{y}_{\mathrm{M}}+\lambda_{\mathrm{S}} p_{\mathrm{SS}} \tilde{y}_{\mathrm{S}}\right)+\left(\lambda_{\mathrm{M}} \alpha_{\mathrm{M}} \tilde{y}_{\mathrm{M}}+\lambda_{\mathrm{S}} \alpha_{\mathrm{S}} \tilde{y}_{\mathrm{S}}\right) \tilde{y}_{\mathrm{M}}-\gamma_{\mathrm{S}} \tilde{y}_{\mathrm{S}}}{\lambda_{\mathrm{M}} \tilde{y}_{\mathrm{M}}+\lambda_{\mathrm{S}} \tilde{y}_{\mathrm{S}}} \\
\frac{d \tilde{z}_{\mathrm{M}}}{d t} & =\frac{\gamma_{\mathrm{M}} \tilde{y}_{\mathrm{M}}}{\lambda_{\mathrm{M}} \tilde{y}_{\mathrm{M}}+\lambda_{\mathrm{S}} \tilde{y}_{\mathrm{S}}}, \\
\frac{d \tilde{z}_{\mathrm{S}}}{d t} & =\frac{\gamma_{\mathrm{S}} \tilde{y}_{\mathrm{S}}}{\lambda_{\mathrm{M}} \tilde{y}_{\mathrm{M}}+\lambda_{\mathrm{S}} \tilde{y}_{\mathrm{S}}} .
\end{aligned}
$$

Let $\tilde{\tau}=\inf \left\{t \geq 0: \tilde{y}_{\mathrm{M}}(t)+\tilde{y}_{\mathrm{S}}(t) \leq 0\right\}$. It is easily verified that $\tilde{\tau}<\infty$. Let $\tilde{\tau}^{(n)}=\inf \{t>$ $\left.0: \tilde{Y}_{\mathrm{M}}^{(n)}(t)+\tilde{Y}_{\mathrm{S}}^{(n)}(t)=0\right\}\left(=A^{(n)}\right)$, so $\tilde{\boldsymbol{X}}^{(n)}\left(\tilde{\tau}^{(n)}\right)$ yields the final outcome of $E^{(n)}$. We seek a central limit theorem for $\tilde{\boldsymbol{X}}^{(n)}\left(\tilde{\tau}^{(n)}\right)$ but first some more notation is required.

Let $\partial \tilde{F}(\tilde{\boldsymbol{x}})=\left[\partial_{j} \tilde{F}_{i}(\tilde{\boldsymbol{x}})\right]$ denote the matrix of first partial derivatives of $\tilde{F}(\tilde{\boldsymbol{x}})$ and, for $0 \leq s \leq$ $t \leq \tilde{\tau}$, let $\tilde{\Phi}(t, s)$ be the solution of the matrix differential equation

$$
\frac{\partial}{\partial t} \tilde{\Phi}(t, s)=\partial \tilde{F}(\tilde{\boldsymbol{x}}(t)) \tilde{\Phi}(t, s), \quad \tilde{\Phi}(s, s)=I,
$$

where $I$ denotes the $5 \times 5$ identity matrix. Let $\tilde{G}(\tilde{\boldsymbol{x}})=\sum_{\boldsymbol{l} \in \Delta} \tilde{\beta}_{\boldsymbol{l}}(\tilde{\boldsymbol{x}}) \boldsymbol{l} \boldsymbol{l}^{\top}$. For $t \in[0, \tilde{\tau}]$, let

$$
\tilde{\boldsymbol{V}}^{(n)}(t)=\sqrt{n}\left(n^{-1} \tilde{\boldsymbol{X}}^{(n)}(t)-\tilde{\boldsymbol{x}}(t)\right),
$$


where, $\tilde{\boldsymbol{X}}^{(n)}(t)=\tilde{\boldsymbol{X}}^{(n)}\left(\tilde{\tau}^{(n)}\right)$ if $t>\tilde{\tau}^{(n)}$, and suppose that $\lim _{n \rightarrow \infty} \sqrt{n}\left(n^{-1} m_{\mathrm{M}}^{(n)}-\mu_{\mathrm{M}}\right)=0$ and $\lim _{n \rightarrow \infty} \sqrt{n}\left(n^{-1} m_{\mathrm{S}}^{(n)}-\mu_{\mathrm{S}}\right)=0$. For $t_{0} \in(0, \tilde{\tau}), \tilde{\boldsymbol{F}}$ is Lipschitz-continuous and $\partial \tilde{F}$ is differentiable in a small neighbourhood of $\left\{\tilde{\boldsymbol{x}}(u): 0 \leq u \leq t_{0}\right\}$. Further, for $\boldsymbol{l} \in \Delta$, $\tilde{\beta}_{\boldsymbol{l}}$ is bounded on any compact subset of $[0,1] \times(0, \infty)^{4}$. Thus the conditions of Theorem 11.2.3 of Ethier and Kurtz [17], are satisfied, so, for any $t_{0} \in(0, \tilde{\tau})$,

$$
\left\{\tilde{\boldsymbol{V}}^{(n)}(t): 0 \leq t \leq t_{0}\right\} \Rightarrow\left\{\tilde{\boldsymbol{V}}(t): 0 \leq t \leq t_{0}\right\}
$$

where $\Rightarrow$ denotes weak convergence in the space of right-continuous functions from $\left[0, t_{0}\right] \rightarrow \mathbb{R}^{5}$ with left limits, endowed with the Skorohod topology, and $\left\{\tilde{\boldsymbol{V}}(t): 0 \leq t \leq t_{0}\right\}$ is a zero-mean Gaussian process with $\tilde{\boldsymbol{V}}(0)=\mathbf{0}$ and covariance function given by

$$
\operatorname{cov}(\tilde{\boldsymbol{V}}(t), \tilde{\boldsymbol{V}}(s))=\int_{0}^{\min (t, s)} \tilde{\Phi}(t, u) \tilde{G}(\tilde{\boldsymbol{x}}(u))[\tilde{\Phi}(s, u)]^{\top} d u
$$

Let $\phi\left(\tilde{x}, \tilde{y}_{\mathrm{M}}, \tilde{y}_{\mathrm{S}}, \tilde{z}_{\mathrm{M}}, \tilde{z}_{\mathrm{S}}\right)=\tilde{y}_{\mathrm{M}}+\tilde{y}_{\mathrm{S}}$, so $\tilde{\tau}^{(n)}=\inf \left\{t>0: \phi\left(\tilde{\boldsymbol{X}}^{(n)}(t)\right) \leq 0\right\}$ and $\tilde{\tau}=\inf \{t>0$ : $\phi(\tilde{\boldsymbol{x}}(t)) \leq 0\}$. Provided its conditions are satisfied, Theorem 11.4.1 of Ethier and Kurtz [17] implies that

$$
\sqrt{n}\left(n^{-1} \tilde{\boldsymbol{X}}^{(n)}\left(\tilde{\tau}^{(n)}\right)-\tilde{\boldsymbol{x}}(\tilde{\tau})\right) \stackrel{D}{\longrightarrow} \tilde{\boldsymbol{V}}(\tilde{\tau})-\frac{\nabla \phi(\tilde{\boldsymbol{x}}(\tilde{\tau})) \tilde{\boldsymbol{V}}(\tilde{\tau})}{\nabla \phi(\tilde{\boldsymbol{x}}(\tilde{\tau})) \tilde{F}(\tilde{\boldsymbol{x}}(\tilde{\tau}))} \tilde{F}(\tilde{\boldsymbol{x}}(\tilde{\tau})) \quad \text { as } \quad n \rightarrow \infty
$$

where $\stackrel{D}{\longrightarrow}$ denotes convergence in distribution. Now, $\nabla \phi(\tilde{\boldsymbol{x}})=(0,1,1,0,0)$, so, using $(5.11)$,

$$
\nabla \phi(\tilde{\boldsymbol{x}}(t)) \tilde{x}(t)-\frac{\gamma_{\mathrm{M}} \tilde{y}_{\mathrm{M}}(t)-\gamma_{\mathrm{S}} \tilde{y}_{\mathrm{S}}(t)}{\lambda_{\mathrm{M}} \tilde{y}_{\mathrm{M}}(t)+\lambda_{\mathrm{S}} \tilde{y}_{\mathrm{S}}(t)} \quad(t \in[0, \tau)) .
$$

Assuming that the limit exists, let $\theta=\lim _{t \rightarrow \tilde{\tau}-}\left[\tilde{y}_{\mathrm{M}}(t) / \tilde{y}_{\mathrm{S}}(t)\right]$. Then, using L'Hôpital's rule,

$$
\begin{aligned}
\theta & =\lim _{t \rightarrow \tilde{\tau}-} \frac{\tilde{y}_{\mathrm{M}}^{\prime}(t)}{\tilde{y}_{\mathrm{S}}^{\prime}(t)} \\
& =\lim _{t \rightarrow \tilde{\tau}-}\left[\frac{\tilde{x}(t)\left(\lambda_{\mathrm{M}} p_{\mathrm{MM}} \tilde{y}_{\mathrm{M}}(t)+\lambda_{\mathrm{S}} p_{\mathrm{SM}} \tilde{y}_{\mathrm{S}}(t)\right)-\gamma_{\mathrm{M}} \tilde{y}_{\mathrm{M}}(t)-\left(\lambda_{\mathrm{M}} \alpha_{\mathrm{M}} \tilde{y}_{\mathrm{M}}(t)+\lambda_{\mathrm{S}} \alpha_{\mathrm{S}} \tilde{y}_{\mathrm{M}}(t)\right) \tilde{y}_{\mathrm{M}}(t)}{\tilde{x}(t)\left(\lambda_{\mathrm{M}} p_{\mathrm{MS}} \tilde{y}_{\mathrm{M}}(t)+\lambda_{\mathrm{S}} p_{\mathrm{SS}} \tilde{y}_{\mathrm{S}}(t)\right)-\gamma_{\mathrm{S}} \tilde{y}_{\mathrm{S}}(t)+\left(\lambda_{\mathrm{M}} \alpha_{\mathrm{M}} \tilde{y}_{\mathrm{M}}(t)+\lambda_{\mathrm{S}} \alpha_{\mathrm{S}} \tilde{y}_{\mathrm{M}}(t)\right) \tilde{y}_{\mathrm{M}}(t)}\right] \\
& =\frac{\tilde{x}(\tilde{\tau})\left(\lambda_{\mathrm{M}} p_{\mathrm{MM}} \theta+\lambda_{\mathrm{S}} p_{\mathrm{SM}}\right)-\gamma_{\mathrm{M}} \theta}{\tilde{x}(\tilde{\tau})\left(\lambda_{\mathrm{M}} p_{\mathrm{MS}} \theta+\lambda_{\mathrm{S}} p_{\mathrm{SS}}\right)-\gamma_{\mathrm{S}}} .
\end{aligned}
$$

Thus, provided $\tilde{x}(\tilde{\tau})\left(\lambda_{\mathrm{M}} p_{\mathrm{MS}} \theta+\lambda_{\mathrm{S}} p_{\mathrm{SS}}\right)-\gamma_{\mathrm{S}} \neq 0, \theta$ satisfies the quadratic equation

$$
\tilde{x}(\tilde{\tau}) \lambda_{\mathrm{M}} p_{\mathrm{MS}} \theta^{2}+\left[x(\tilde{\tau})\left(\lambda_{\mathrm{S}} p_{\mathrm{SS}}-\lambda_{\mathrm{M}} p_{\mathrm{MM}}\right)+\gamma_{\mathrm{M}}-\gamma_{\mathrm{S}}\right] \theta-\tilde{x}(\tilde{\tau}) \lambda_{\mathrm{S}} p_{\mathrm{SM}}=0 .
$$

Suppose that $p_{\mathrm{MS}}>0$ and $p_{\mathrm{SM}}>0$. Then the roots of (5.20) have opposite signs and, since $\tilde{y}_{\mathrm{M}}(t)$ and $\tilde{y}_{\mathrm{S}}(t)$ are each nonnegative for $t \in[0, \tilde{\tau}), \theta$ is given by the positive root. Letting 
$t \rightarrow \tilde{\tau}-$ in $(5.18)$ now yields

$$
\begin{aligned}
\nabla \phi(\tilde{\boldsymbol{x}}(\tilde{\tau})) \tilde{x}(\tilde{\tau})-\frac{\gamma_{\mathrm{M}} \theta-\gamma_{\mathrm{S}}}{\lambda_{\mathrm{M}} \theta+\lambda_{\mathrm{S}}} \\
=\frac{(1+\theta)\left[\tilde{x}(\tilde{\tau})\left(\lambda_{\mathrm{M}} p_{\mathrm{MS}} \theta+\lambda_{\mathrm{S}} p_{\mathrm{SS}}\right)-\gamma_{\mathrm{S}}\right]}{\lambda_{\mathrm{M}} \theta+\lambda_{\mathrm{S}}}
\end{aligned}
$$

using (5.19). Now $\nabla \phi(\tilde{\boldsymbol{x}}(\tilde{\tau})) \leq 0$ by the definition of $\tilde{\tau}$, so (5.21) implies that $\nabla \phi(\tilde{\boldsymbol{x}}(\tilde{\tau}))<0$, since $\theta>0$ and $\tilde{x}(\tilde{\tau})\left(\lambda_{\mathrm{M}} p_{\mathrm{MS}} \theta+\lambda_{\mathrm{S}} p_{\mathrm{SS}}\right)-\gamma_{\mathrm{S}} \neq 0$.

In addition to $\nabla \phi(\tilde{\boldsymbol{x}}(\tilde{\tau}))<0$, Theorem 11.4.1 of Ethier and Kurtz [17] requires that (5.15) holds for some $t_{0}>\tilde{\tau}$. This requires extending the transition rate functions $\tilde{\beta}_{\boldsymbol{l}}(\boldsymbol{l} \in \Delta)$, defined at $(5.11)$, so that $\tilde{\boldsymbol{x}}(t)$ is defined on $\left[0, t_{0}\right)$ for some $t_{0}>\tilde{\tau}$, with the conditions of Theorem 11.2.3 of Ethier and Kurtz [17] still being satisfied, in particular so that $\tilde{F}$ is Lipschitz-continuous in a neighbourhood of $\left\{\tilde{\boldsymbol{x}}(t): 0 \leq t \leq t_{0}\right\}$, and this does not seem possible. However, assuming that (5.17) still holds, it follows that

$$
\sqrt{n}\left(n^{-1} \tilde{\boldsymbol{X}}^{(n)}\left(\tilde{\tau}^{(n)}\right)-\tilde{\boldsymbol{x}}(\tilde{\tau})\right) \stackrel{D}{\longrightarrow} N\left(\mathbf{0}, B \tilde{\Sigma}(\tilde{\tau}) B^{\top}\right) \quad \text { as } \quad n \rightarrow \infty,
$$

where $\tilde{\Sigma}(\tilde{\tau})=\operatorname{cov}(\tilde{\boldsymbol{V}}(\tilde{\tau}), \tilde{\boldsymbol{V}}(\tilde{\tau}))$ and

$$
B=I-\frac{\tilde{F}(\tilde{\boldsymbol{x}}(\tilde{\tau}))(0,1,1,0,0)}{(0,1,1,0,0) \tilde{F}(\tilde{\boldsymbol{x}}(\tilde{\tau}))}
$$

Note that (5.14) and (5.16) imply that $\tilde{\Sigma}(t)=\operatorname{cov}(\tilde{\boldsymbol{V}}(t), \tilde{\boldsymbol{V}}(t))(0 \leq t \leq \tilde{\tau})$ satisfies

$$
\frac{d \tilde{\Sigma}}{d t}=\tilde{G}(\tilde{\boldsymbol{x}})+\partial \tilde{F}(\tilde{\boldsymbol{x}}) \tilde{\Sigma}+\tilde{\Sigma}[\partial \tilde{F}(\tilde{\boldsymbol{x}})]^{\top}, \quad \tilde{\Sigma}(0)=0 .
$$

Thus, in principle, $\tilde{\Sigma}(\tilde{\tau})$ can be computed by solving numerically the differential equations (5.13) and (5.23) simultaneously. However, in practice we have found that this procedure is not very accurate in some examples and much better results are obtained by first transforming 5.13) and (5.23) back into the original time domain. Thus,

$$
\left.\frac{d \Sigma}{d t}=\left(\lambda_{\mathrm{M}} y_{\mathrm{M}}+\lambda_{\mathrm{S}} y_{\mathrm{S}}\right)(\tilde{G}(\boldsymbol{x})+\partial \tilde{F}(\boldsymbol{x}) \Sigma+\Sigma \partial \tilde{F}(\boldsymbol{x})]^{\top}\right), \quad \Sigma(0)=0,
$$

is solved numerically simultaneously with (3.6), where $\boldsymbol{x}(0)=\left(1, \mu_{\mathrm{M}}, \mu_{\mathrm{S}}, 0,0\right)^{\top}$, whence $(\boldsymbol{x}(\tilde{\tau}), \tilde{\Sigma}(\tilde{\tau}))=$ $(\boldsymbol{x}(\infty), \Sigma(\infty))$.

Clearly the above argument is heuristic in places. However, the assertions concerning the limit $\theta$ are supported by all of our numerical studies, as is the central limit theorem (5.22) for the final outcome of the epidemic. 


\section{Discussion}

The IEDS model combines the possibility that the severity depends on who one is infected by, as in the IDS model (Ball and Britton [4]), and that it is possible to become severely infected if additionally exposed to the disease, as considered in the EDS model (Ball and Britton [9]). In that sense the model is now quite flexible and realistic. Of course, there are several aspects in which the model can be made more realistic. The assumption of homogeneous mixing could be relaxed, for example by introducing households (cf. Ball and Becker [21]). Another generalisation of interest would be to allow for different types of individuals, e.g. age cohorts, having different transmission rates between different types of individuals. The model can also be generalised by having more than two degrees of severity, and by allowing for an arbitrary distribution of the infectious period.

From a statistical point of view it would of course be interesting to derive parameter estimators and estimate model parameters for specific diseases. In particular it would be interesting to study if severe cases typically appear directly upon infection or when additionally exposed. For such estimation to be possibly it will be necessary to have some type of temporal data rather than only final size data.

A specific example of the mild severity is for diseases having asymptomatic cases. Usually such cases shed less infectious matter per time unit, but on the other hand they may be infectious for longer, since severe cases typically stay home once symptoms appear, thus effectively ending their infectious period. This can be reflected in the present model by having $\lambda_{\mathrm{M}}<\lambda_{\mathrm{S}}$ and $\gamma_{\mathrm{M}}<\gamma_{\mathrm{S}}$. It could very well be that the total expected infectivity shed by a mild case may exceed that of a severe case $\left(\lambda_{\mathrm{M}} / \gamma_{\mathrm{M}}>\lambda_{\mathrm{S}} / \gamma_{\mathrm{S}}\right)$, but this is allowed for in the present model.

The vaccine response model treated in the present paper is quite general in that it allows infectivity reduction in two ways: lower infectivity and/or shorter infectious period. It would be interesting to study this general form of response model also for other epidemic models. In work not presented here owing to space considerations, the authors consider this response model for a simple SIS (susceptible $\rightarrow$ infective $\rightarrow$ susceptible) epidemic in a partially vaccinated community; for the parameter values considered, the overall endemic level increases with $\theta$.

\section{Appendix A Properties of deterministic final outcome}

Suppose first that $\mu>0$ and recall that $R_{\mathrm{S}}>R_{\mathrm{M}}$. For fixed $z_{\mathrm{S}} \in[0, \mu]$, define functions $f_{z_{\mathrm{S}}}:[0, \infty) \rightarrow[0, \infty)$ and $g_{z_{\mathrm{S}}}:[0, \infty) \rightarrow[0, \infty)$ by $f_{z_{\mathrm{S}}}\left(z_{\mathrm{M}}\right)=\exp \left(-\left(R_{\mathrm{M}} z_{\mathrm{M}}+R_{\mathrm{S}} z_{\mathrm{S}}\right)\right)$ and $g_{z_{\mathrm{S}}}\left(z_{\mathrm{M}}\right)=1+\mu-z_{\mathrm{S}}-z_{\mathrm{M}}$. For $z_{\mathrm{z}} \in[0, \mu]$, the solutions of $f_{z_{\mathrm{S}}}\left(z_{\mathrm{M}}\right)=g_{z_{\mathrm{S}}}\left(z_{\mathrm{M}}\right)$ give the values of $\hat{z}_{\mathrm{M}}$ so that $\left(\hat{z}_{\mathrm{M}}, \hat{z}_{\mathrm{S}}\right)$ satisfies $(3.10)$ with $\hat{z}_{\mathrm{S}}=z_{\mathrm{S}}$. It is easily seen that $f_{z_{\mathrm{S}}}(0)<g_{z_{\mathrm{S}}}(0)$ if $z_{\mathrm{S}}<z_{\mathrm{S}}^{*}, f_{z_{\mathrm{S}}}(0)=g_{z_{\mathrm{S}}}(0)$ if $z_{\mathrm{S}}=z_{\mathrm{S}}^{*}$ and $f_{z_{\mathrm{S}}}(0)>g_{z_{\mathrm{S}}}(0)$ if $z_{\mathrm{S}}>z_{\mathrm{S}}^{*}$, where $z_{\mathrm{S}}^{*}$ is the positive solution of $1+\mu-z=\exp \left(-R_{\mathrm{S}} z\right)$. The function $f_{z_{\mathrm{S}}}$ is convex and strictly decreasing, and 
it is easily seen that, if $z_{\mathrm{S}}<z_{\mathrm{S}}^{*}$, then $f_{z_{\mathrm{S}}}-g_{z_{\mathrm{S}}}$ has a unique root in $[0,1+\mu]$. Thus, $h\left(z_{\mathrm{S}}\right)$ is well defined for $z_{\mathrm{S}} \in\left[0, z_{\mathrm{S}}^{*}\right)$. Now, 0 is clearly a root of $f_{z_{\mathrm{S}}^{*}}-g_{z_{\mathrm{S}}^{*}}$ and considering the first derivatives of $f_{z_{\mathrm{S}}^{*}}$ and $g_{z_{\mathrm{S}}^{*}}$ at the origin shows that it is the only positive root if and only if $R_{\mathrm{M}} \exp \left(-R_{\mathrm{S}} z_{\mathrm{S}}^{*}\right) \leq 1$. Recall that $1+\mu-z_{\mathrm{S}}^{*}=\exp \left(-R_{\mathrm{S}} z_{\mathrm{S}}^{*}\right)$. Hence, 0 is the only positive root of $f_{z_{\mathrm{S}}^{*}}-g_{z_{\mathrm{S}}^{*}}$ if and only if

$$
z_{\mathrm{S}}^{*} \geq 1+\mu-\frac{1}{R_{\mathrm{M}}} .
$$

The inequality (A.1) is clearly satisfied if $R_{\mathrm{M}} \leq(1+\mu)^{-1}$, so assume that $R_{\mathrm{M}}>(1+\mu)^{-1}$. Since $R_{\mathrm{S}}>R_{\mathrm{M}},\left(\right.$ A.1) is satisfied if $z_{\mathrm{S}}^{*} \geq 1+\mu-\frac{1}{R_{\mathrm{S}}}$. Let $f:[0, \infty) \rightarrow[0, \infty)$ and $g:[0, \infty) \rightarrow[0, \infty)$ be defined by $f(z)=\exp \left(-R_{\mathrm{S}} z\right)$ and $g(z)=1+\mu-z$. Then $z_{\mathrm{S}}^{*}$ is the positive root of $f-g$ and $z_{\mathrm{S}}^{*} \geq 1+\mu-\frac{1}{R_{\mathrm{S}}}$ if and only if $f\left(1+\mu-\frac{1}{R_{\mathrm{S}}}\right) \leq g\left(1+\mu-\frac{1}{R_{\mathrm{S}}}\right)$. Elementary algebra shows that this last condition is equivalent to $\exp \left(R_{\mathrm{S}}(1+\mu)-1\right) \geq R_{\mathrm{S}}$, which clearly holds as $R_{\mathrm{S}}>R_{\mathrm{M}}>(1+\mu)^{-1}$. Thus, 0 is the only positive root of $f_{z_{\mathrm{S}}^{*}}-g_{z_{\mathrm{S}}^{*}}$ and $h\left(z_{\mathrm{S}}\right)$ is well defined for $z_{\mathrm{S}} \in\left[0, z_{\mathrm{S}}^{*}\right.$. Let $f_{z_{\mathrm{S}}}^{\prime}$ denote the first derivative of $f_{z_{\mathrm{S}}}$. Then $f_{z_{\mathrm{S}}}^{\prime}(0)$ increases with $z_{\mathrm{S}}$ and it is easily shown that $f_{z_{\mathrm{S}}}-g_{z_{\mathrm{S}}}$ has no positive root when $z_{\mathrm{S}}>z_{\mathrm{S}}^{*}$. Thus, (3.10) has no solution in the first quadrant with $\hat{z}_{\mathrm{S}}>z_{\mathrm{S}}^{*}$.

The following lemma is useful in proving the stated properties of the function $h$.

Lemma A.1 Suppose that $\left(z_{\mathrm{M}}, z_{\mathrm{S}}\right)$ is a solution of (3.10) in the first quadrant and $R_{\mathrm{M}}<R_{\mathrm{S}}$. Then,

$$
R_{\mathrm{M}}\left(1+\mu-z_{\mathrm{M}}-z_{\mathrm{S}}\right)<1
$$

Proof: The result is immediate if $R_{\mathrm{M}} \leq(1+\mu)^{-1}$, since $(0,0)$ is not a solution of (3.10). A similar argument to the above shows that (A.1) holds with strict inequality, whence (A.2) holds if $z_{\mathrm{S}}=z_{\mathrm{S}}^{*}$. Thus, suppose that $R_{\mathrm{M}}>(1+\mu)^{-1}$ and $z_{\mathrm{S}} \in\left[0, z_{\mathrm{S}}^{*}\right)$. Condition (A.2) is equivalent to $z_{\mathrm{M}}>1+\mu-z_{\mathrm{S}}-R_{\mathrm{M}}^{-1}$, which holds if $f_{z_{\mathrm{S}}}\left(1+\mu-z_{\mathrm{S}}-R_{\mathrm{M}}^{-1}\right)<g_{z_{\mathrm{S}}}\left(1+\mu-z_{\mathrm{S}}-R_{\mathrm{M}}^{-1}\right)$. After rearranging, the latter condition is equivalent to $\exp \left(\left(R_{\mathrm{S}}-R_{\mathrm{M}}\right) z_{\mathrm{S}}\right) \exp \left(R_{\mathrm{M}}(1+\mu)-1\right)>$ $R_{\mathrm{M}}$. Now $\exp \left(\left(R_{\mathrm{S}}-R_{\mathrm{M}}\right) z_{\mathrm{S}}\right) \geq 1$, as $R_{\mathrm{S}}>R_{\mathrm{M}}$, and $\exp \left(R_{\mathrm{M}}(1+\mu)-1\right)>R_{\mathrm{M}}$, as $R_{\mathrm{M}}>$ $(1+\mu)^{-1}$, and (A.2) follows.

Differentiating the equation

$$
1+\mu-z_{\mathrm{S}}-z_{\mathrm{M}}=\exp \left(-\left(R_{\mathrm{M}} z_{\mathrm{M}}+R_{\mathrm{S}} z_{\mathrm{S}}\right)\right)
$$

implicitly with respect to $z_{\mathrm{S}}$ and rearranging yields

$$
\frac{d z_{\mathrm{M}}}{d z_{\mathrm{S}}}=-\frac{1-R_{\mathrm{S}}\left(1+\mu-z_{\mathrm{S}}-z_{\mathrm{M}}\right)}{1-R_{\mathrm{M}}\left(1+\mu-z_{\mathrm{S}}-z_{\mathrm{M}}\right)}
$$

whence

$$
\frac{d\left(z_{\mathrm{M}}+z_{\mathrm{S}}\right)}{d z_{\mathrm{S}}}=\frac{\left(R_{\mathrm{S}}-R_{\mathrm{M}}\right)\left(1+\mu-z_{\mathrm{S}}-z_{\mathrm{M}}\right)}{1-R_{\mathrm{M}}\left(1+\mu-z_{\mathrm{S}}-z_{\mathrm{M}}\right)}
$$


Now $1-R_{\mathrm{M}}\left(1+\mu-z_{\mathrm{S}}-z_{\mathrm{M}}\right)>0$ by Lemma A.1, $z_{\mathrm{S}}+z_{\mathrm{M}}<1+\mu$ and $R_{\mathrm{M}}<R_{\mathrm{S}}$, so $\frac{d\left(z_{\mathrm{M}}+z_{\mathrm{S}}\right)}{d z_{\mathrm{S}}}>0$, i.e. $h\left(z_{\mathrm{S}}\right)+z_{\mathrm{S}}$ strictly increases with $z_{\mathrm{S}}$ for $z_{\mathrm{S}} \in\left(0, z_{\mathrm{S}}^{*}\right)$.

Differentiating (A.4) with respect to $z_{\mathrm{S}}$ and rearranging yields

$$
\begin{aligned}
\frac{d^{2} z_{\mathrm{M}}}{d z_{\mathrm{S}}^{2}} & =-\frac{R_{\mathrm{S}}-R_{\mathrm{M}}}{\left(1-R_{\mathrm{M}}\left(1+\mu-z_{\mathrm{S}}-z_{\mathrm{M}}\right)\right)^{2}} \frac{d\left(z_{\mathrm{M}}+z_{\mathrm{S}}\right)}{d z_{\mathrm{S}}} \\
& <0,
\end{aligned}
$$

for $z_{\mathrm{S}} \in\left(0, z_{\mathrm{S}}^{*}\right)$, since $R_{\mathrm{M}}<R_{\mathrm{S}}$ and $\frac{d\left(z_{\mathrm{M}}+z_{\mathrm{S}}\right)}{d z_{\mathrm{S}}}>0$. Thus $h$ is concave on $\left(0, z_{\mathrm{S}}^{*}\right)$.

Note that $h:\left[0, z_{\mathrm{S}}^{*}\right] \rightarrow[0,1+\mu)$ is injective if and only if $h^{\prime}(0) \leq 0$. Recall that $h(0)=z_{\mathrm{M}}^{*}$, where $z_{\mathrm{M}}^{*}$ is the positive root of $1+\mu-z=\exp \left(-R_{\mathrm{M}} z\right)$. Then, from (A.4) and using Lemma A.1, $h^{\prime}(0) \leq 0$ if and only if $z_{\mathrm{M}}^{*} \geq 1+\mu-R_{\mathrm{S}}^{-1}$. Note that $z_{\mathrm{M}}^{*}$ is given by the positive root of $f_{0}-g_{0}$, so a necessary and sufficient condition for $z_{\mathrm{M}}^{*} \geq 1+\mu-R_{\mathrm{S}}^{-1}$ is that $f_{0}\left(1+\mu-R_{\mathrm{S}}^{-1}\right)<g_{0}\left(1+\mu-R_{\mathrm{S}}^{-1}\right)$, which is equivalent to

$$
R_{\mathrm{M}}\left(R_{\mathrm{S}}(1+\mu)-1\right) \geq R_{\mathrm{S}} \log R_{\mathrm{S}}
$$

Let $R_{\mathrm{M}}^{*}=\max \left(R_{\mathrm{S}} \log R_{\mathrm{S}} /\left[R_{\mathrm{S}}(1+\mu)-1\right], 0\right)$. Then it is easily seen that, if $R_{\mathrm{S}} \geq(1+\mu)^{-1}$ then $R_{\mathrm{M}}^{*}<R_{\mathrm{S}}$ and (A.5) is satisfied if and only if $R_{\mathrm{M}} \geq R_{\mathrm{M}}^{*}$. If $R_{\mathrm{S}}<(1+\mu)^{-1}$ then $\exp \left(-\left(1-R_{\mathrm{S}}(1+\mu)\right)\right)>R_{\mathrm{S}}(1+\mu)>R_{\mathrm{S}}$, so $\frac{\log R_{\mathrm{S}}}{R_{\mathrm{S}}(1+\mu)-1}>1$ and (A.5) is not satisfied for any $R_{\mathrm{M}} \in\left[0, R_{\mathrm{S}}\right)$. Thus, if $R_{\mathrm{S}} \geq(1+\mu)^{-1}$, then $h:\left[0, z_{\mathrm{S}}^{*}\right] \rightarrow[0,1+\mu)$ is injective if and only if $R_{\mathrm{M}} \geq R_{\mathrm{M}}^{*}$, while, if $R_{\mathrm{S}}<(1+\mu)^{-1}$, then $h$ is not injective for any $R_{\mathrm{M}} \in\left[0, R_{\mathrm{S}}\right)$.

Suppose now that $\mu=0$ and note that in this case $(0,0)$ is always a solution of (3.10). Now, since $R_{\mathrm{M}}<R_{\mathrm{S}}$, for $z_{\mathrm{M}}, z_{\mathrm{S}} \geq 0$,

$$
\exp \left(\left(-\left(R_{\mathrm{M}} z_{\mathrm{M}}+R_{\mathrm{S}} z_{\mathrm{S}}\right)\right) \geq \exp \left(-R_{\mathrm{S}}\left(z_{\mathrm{M}}+z_{\mathrm{S}}\right)\right) \geq 1-R_{\mathrm{S}}\left(z_{\mathrm{M}}+z_{\mathrm{S}}\right),\right.
$$

with strict inequality unless $\left(z_{\mathrm{M}}, z_{\mathrm{S}}\right)=(0,0)$. Hence, $(0,0)$ is the only solution of $(3.10)$ in the first quadrant if $R_{\mathrm{S}} \leq 1$. If $R_{\mathrm{S}}>1$, similar arguments to the above yield the properties of the solutions of (3.10) stated in Section 3.3. The main difference is that now zero is always a root of $f_{0}-g_{0}$ and there is a strictly positive root if and only $R_{\mathrm{M}}>1$. Thus $z_{\mathrm{M}}^{*}>0$ if and only if $R_{\mathrm{M}}>1$.

\section{Appendix B Proof of stochastic comparison (3.12)}

We describe first how a realisation of the stochastic IEDS model $E^{(n)}$ can be obtained by adapting the construction of Sellke [18]. Label the individuals as in Section 5.1. Let $L_{1}, L_{2}, \ldots, L_{n}$ be $\operatorname{Exp}(1)$ (i.e. unit-mean exponential) random variables (the "resistances to infection" for the initial susceptibles), $L_{-m_{\mathrm{M}}+1}^{\prime}, L_{-m_{\mathrm{M}}+2}^{\prime}, \ldots, L_{n}^{\prime}$ be $\operatorname{Exp}(1)$ random variables (the "resistances to switching to severe" for the initial mild infectives and susceptibles), 
$X_{-\left(m_{\mathrm{M}}+m_{\mathrm{S}}\right)+1}, X_{-\left(m_{\mathrm{M}}+m_{\mathrm{S}}\right)+2}, \ldots, X_{n}$ be $\operatorname{Exp}(1)\left(X_{i}\right.$ is used to determinine the length of the infectious period(s) for individual $i$ if it becomes infected) and, finally, let $U_{1}, U_{2}, \ldots, U_{n}$ be random variables that are uniformly distributed on the interval $(0,1)\left(U_{i}\right.$ is used to determine whether individual $i$ becomes a mild or severe infective if it becomes infected). All the above random variables are mutually independent.

We now construct a realisation of $E^{(n)}$ using these random variables. We use the same notation as in Section 5.1 but suppress the explicit dependence on $n$. Start at time $t=0$ and set $X(0)=$ $n, Y_{\mathrm{M}}(0)=m_{\mathrm{M}}$ and $Y_{\mathrm{S}}(0)=m_{\mathrm{S}}$. For $i=-\left(m_{\mathrm{M}}+m_{\mathrm{S}}\right)+1,-\left(m_{\mathrm{M}}+m_{\mathrm{S}}\right)+2, \ldots,-m_{\mathrm{M}}$, the initial severe infective $i$ recovers and becomes immune at time $\gamma_{\mathrm{S}}^{-1} X_{i}$. For $j=-m_{\mathrm{M}}+1,-m_{\mathrm{M}}+$ $2, \ldots, 0$, the initial mild infective $j$ recovers and becomes immune at time $\gamma_{\mathrm{M}}^{-1} X_{j}$, unless it has switched to severe prior to this time. For any $t \geq 0$, we define the accumulated infection pressure $A(t)$ by $A(t)=n^{-1} \int_{0}^{t} \lambda_{\mathrm{M}} Y_{\mathrm{M}}(s)+\lambda_{\mathrm{S}} Y_{\mathrm{S}}(s) d s$ and the accumulated switching pressure $W(t)$ by $W(t)=n^{-1} \int_{0}^{t} \lambda_{\mathrm{M}} \alpha_{\mathrm{M}} \max \left(Y_{\mathrm{M}}(s)-1,0\right)+\lambda_{\mathrm{S}} Y_{\mathrm{S}}(s) \alpha_{\mathrm{S}} d s$. (The term $\max \left(Y_{\mathrm{M}}(s)-1,0\right)$ arises because a mild infective cannot reinfect itself, cf. the factor $\left(i_{\mathrm{M}}-1\right)$ in (5.10).) Move forward in time (from $t=0$ ) until either (i) the accumulated infection pressure $A(t)$ reaches the resistance of any susceptible, or (ii) the accumulated switching pressure $W(t)$ reaches the switching resistance of a mild infective, or (iii) the infectious period of any mild or severe infective terminates. If (i) occurs, say $A(t)$ reaches $L_{i}$, then the number of susceptibles $X(t)$ is reduced by one; individual $i$ becomes a mild infective and $Y_{\mathrm{M}}(t)$ is increased by one if

$$
U_{i} \leq \frac{\lambda_{\mathrm{M}} Y_{\mathrm{M}}(t-) p_{\mathrm{MM}}+\lambda_{\mathrm{S}} Y_{\mathrm{S}}(t-) p_{\mathrm{SM}}}{\lambda_{\mathrm{M}} Y_{\mathrm{M}}(t-)+\lambda_{\mathrm{S}} Y_{\mathrm{S}}(t-)}
$$

otherwise $i$ becomes a severe infective and $Y_{\mathrm{S}}(t)$ is increased by one. If (ii) occurs, say $W(t)$ reaches $L_{j}^{\prime}$, then individual $j$ switches from being mildly to severely infected, $Y_{\mathrm{M}}(t)$ is decreased by one and $Y_{\mathrm{S}}(t)$ is increased by one. Suppose that this switch occurs $\tau_{j}$ units of time after $j$ was first infected. Then $j$ 's severe infectious period has length given by $\gamma_{\mathrm{S}}^{-1}\left(X_{j}-\gamma_{\mathrm{M}} \tau_{j}\right)$. (Note that the lack-of-memory property of the exponential distribution ensures that the length of $j$ 's severe infectious period is exponentially distributed with mean $\gamma_{\mathrm{S}}^{-1}$.) If (iii) occurs, then either $Y_{\mathrm{M}}(t)$ or $Y_{\mathrm{S}}(t)$ is reduced by one, as appropriate, and the relevant individual is removed. Continue moving forward in time, updating the variables as above, until there is no infective of either type in the population, at which point the epidemic terminates. It is easily verified, using the lack-of-memory property of the exponential distribution, that this construction yields a process that is probabilistically indistinguishable from the IEDS model described in Section 2.1.

Consider an individual, $i$ say, in the above epidemic and suppose that $i$ becomes mildly infected and switches to being severely infected $\tau_{i}$ units of time later, so $\tau_{i}<\gamma_{\mathrm{M}}^{-1} X_{i}$. Let $T_{i}^{P}$ denote the total infectious pressure $i$ exerts on any given individual during its entire infectious career. Then, $T_{i}^{P}=n^{-1} T_{P}\left(X_{i}, \tau_{i}\right)$, where

$$
\begin{aligned}
T_{P}(x, \tau) & =\lambda_{\mathrm{M}} \tau+\lambda_{\mathrm{S}}\left(x-\gamma_{\mathrm{M}} \tau\right) \gamma_{\mathrm{S}}^{-1} \\
& =R_{\mathrm{S}} x+\gamma_{\mathrm{M}} \tau\left(R_{\mathrm{M}}-R_{\mathrm{S}}\right) \quad\left(0 \leq \tau<\gamma_{\mathrm{M}}^{-1} x\right) .
\end{aligned}
$$


Note that if $i$ becomes a severe case when first infected then $T_{i}^{P}=n^{-1} T_{P}\left(X_{i}, 0\right)$, and if $i$ becomes a mild case when first infected and never switches to being severe then $T_{i}^{P}=$ $n^{-1} T_{P}\left(X_{i}, \gamma_{\mathrm{M}}^{-1} X_{i}\right)$.

The random variables $L_{1}, L_{2}, \ldots, L_{n}$ and $X_{-\left(m_{\mathrm{M}}+m_{\mathrm{S}}\right)+1}, X_{-\left(m_{\mathrm{M}}+m_{\mathrm{S}}\right)+2}, \ldots, X_{n}$ can also be used to construct a realisation of a general stochastic epidemic with infection parameter $\lambda_{\mathrm{S}}$ and removal rate $\gamma_{\mathrm{S}}, G S E^{(n)}\left(\lambda_{\mathrm{S}}, \gamma_{\mathrm{S}}\right)$ say, by setting $\alpha_{\mathrm{M}}=\alpha_{\mathrm{S}}=\infty$ in the above construction. Similarly, a realisation of a general stochastic epidemic with infection parameter $\lambda_{\mathrm{M}}$ and removal rate $\gamma_{\mathrm{M}}, G S E^{(n)}\left(\lambda_{\mathrm{M}}, \gamma_{\mathrm{M}}\right)$ say, is obtained by setting $\alpha_{\mathrm{M}}=\alpha_{\mathrm{S}}=0$, changing the $m_{\mathrm{S}}$ initial severe infectives to mild infectives and forcing all new infections to be mild cases. Moreover, in $G S E^{(n)}\left(\lambda_{\mathrm{S}}, \gamma_{\mathrm{S}}\right)$, the total infectious pressure exerted by $i$, if it becomes infected, is given by $n^{-1} T_{P}\left(X_{i}, 0\right)$, while in $G S E^{(n)}\left(\lambda_{\mathrm{M}}, \gamma_{\mathrm{M}}\right)$ it is given by $n^{-1} T_{P}\left(X_{i}, \gamma_{\mathrm{M}}^{-1} X_{i}\right)$.

Suppose that $R_{\mathrm{M}} \leq R_{\mathrm{S}}$. Then it is immediate from (B.1) that $T_{P}(x, \tau)$ is decreasing in $\tau$ for each fixed $x>0$. It follows that for any given individual, the total infectious pressure it exerts if it becomes infected in the IEDS epidemic $E^{(n)}$ is bounded below by that for $G S E^{(n)}\left(\lambda_{\mathrm{M}}, \gamma_{\mathrm{M}}\right)$ and above by that for $G S E^{(n)}\left(\lambda_{\mathrm{S}}, \gamma_{\mathrm{S}}\right)$. It then follows that, since the resistances to infection $L_{1}, L_{2}, \ldots, L_{n}$ are the same for the three epidemics, the total size of $E^{(n)}$ is bounded below and above by those of $G S E^{(n)}\left(\lambda_{\mathrm{M}}, \gamma_{\mathrm{M}}\right)$ and $G S E^{(n)}\left(\lambda_{\mathrm{S}}, \gamma_{\mathrm{S}}\right)$, respectively. Thus (3.12) holds with $Z_{\min }$ and $Z_{\max }$ corresponding to $G S E^{(n)}\left(\lambda_{\mathrm{M}}, \gamma_{\mathrm{M}}\right)$ and $G S E^{(n)}\left(\lambda_{\mathrm{S}}, \gamma_{\mathrm{S}}\right)$, respectively. A similar argument holds if $R_{\mathrm{M}} \geq R_{\mathrm{S}}$, except now $T_{P}(x, \tau)$ is decreasing in $\tau$ for each fixed $x>0$, so the bounding general stochastic epidemics are interchanged.

\section{Acknowledgements}

This research was supported by the Swedish Research Council, The Leverhulme Trust and the UK Engineering and Physical Sciences Reserach Council (under grants EP/F03234X/1 and $\mathrm{EP} / \mathrm{E} 038670 / 1)$.

\section{References}

[1] Mangada, M.N.M. and Igarashi, A. (1998) Molecular and in vitro analysis of eight dengue type 2 viruses isolated from patients exhibiting different disease severities. Virology 244, 458-466.

[2] Morley, D.C. and Aaby, P. (1997) Managing measles: size of infecting dose may be important. BMJ 314, 1692.

[3] Parang, N.M. and Archana, C. (2004) Varicella. eMedicine September 15, 2004. http://www.emedicine.com/ped/topic2385.htm 
[4] Ball F.G.and Britton, T. (2007): An epidemic model with infector-dependent severity. Adv. Appl. Prob., 39(4).

[5] Bailey N. T. J. (1975). The Mathematical Theory of Infectious Diseases and its Applications. London: Griffin.

[6] W.S. Kendall, I.W. Saunders, Epidemics in competition II: the general epidemic, J. R. Statist. Soc. B 45 (1983) 238-244.

[7] G.-P. Scalia-Tomba, A. Svensson, Competing epidemics in closed populations, Research Report 2001:8, Department of Mathematical Statistics, Stockholm University.

[8] G.-P. Scalia-Tomba, Epidemic models, multi-strain, in: P. Armitage, N. Colton (Eds.), Encylopedia of Biostatistics, 2nd. Edition, Wiley, Chichester, pp. 1672-1674.

[9] Ball F.G.and Britton T. (2005): An epidemic model with exposure-dependent severities. J. Appl. Prob.,42, 932-949.

[10] Becker, N.G. and Starczak (1998). The effect of random vaccine response on the vaccination coverage required to prevent epidemics. Math. Biosci. 154, 117-135.

[11] Becker, N. G., Britton, T. and O'Neill P. D. (2006) Estimating vaccine effects from studies of outbreaks in household pairs. Statistics in Medicine 25, 1079-1093.

[12] P.G. Smith, L.C. Rodrigues, P.E.M. Fine, Assessment of the protective efficacy of vaccines against common diseases using case-control and cohort studies, Int. J. Epidemiol. 13 (1984) $87-93$.

[13] M.E. Halloran, M. Haber, I.M. Longini, Interpretation and estimation of vaccine efficacy under heterogeneity, Amer. J. Epidemiol. 136 (1992) 328-343.

[14] F. G. Ball, O. D. Lyne, Optimal vaccination schemes for epidemics among a population of households, with application to variola minor in Brazil, Stat. Methods Med. Res. 15 (2006) 481-497.

[15] H. Heesterbeek, K. Dietz, The concept of $R_{0}$ in epidemic theory, Statistica Neerlandica 50 (1996) 89-110.

[16] Haccou, P., Jagers, P. and Vatutin, V. A. (2005) Branching Processes: Variation, Growth, and Extinction of Populations. Cambridge University Press, Cambridge.

[17] Ethier, S. N. and Kurtz, T. G. (1986) Markov Processes: Characterization and Convergence. Wiley, New York.

[18] T. Sellke, On the asymptotic distribution of the size of a stochastic epidemic, J. Appl. Prob. 20 (1983) 390-394. 
[19] D. Greenhalgh, O. Diekmann, M.C.M. de Jong, Subcritical endemic steady states in mathematical models for animal infections with incomplete immunity, Math. Biosci. 165 (2000) 1-25.

[20] P. van den Driessche, J. Watmough, Reproduction numbers and sub-threshold endemic equilibria for compartmental models of disease transmission, Math. Biosci. 180 (2002) $29-48$.

[21] Ball, F.G. and Becker, N.G. (2006) Control of transmission with two types of infection. Math. Biosci. 200, 170-187.

[22] Ball, F. G. and Donnelly, P. J. (1995). Strong approximations for epidemic models. Stoch. Proc. Appl. 55, 1-21.

[23] Athreya, K. B. and Ney, P. E. (1972) Branching Processes. Springer-Verlag, Berlin.

[24] Jagers, P. (1991) The growth and stabilization of populations. Statist. Sci. 6, 269-283. 